The Hooley-Huxley contour method for problems in number fields II: factorization and divisibility

Coleman, M.D.

2002

MIMS EPrint: 2006.127

Manchester Institute for Mathematical Sciences

School of Mathematics

The University of Manchester

\footnotetext{
Reports available from: http://eprints.maths.manchester.ac.uk/

And by contacting: The MIMS Secretary

School of Mathematics

The University of Manchester

Manchester, M13 9PL, UK
} 


\title{
The Hooley-Huxley Contour Method for Problems in Number Fields II: Factorization and Divisibility
}

\author{
M D Coleman
}

\section{Introduction}

Let $L$ be a Galois extension of the number field $K$. Set $n=n_{K}=$ $\operatorname{deg} K / \mathbb{Q}, n_{L}=\operatorname{deg} L / \mathbb{Q}$ and $n_{L / K}=\operatorname{deg} L / K$. Let $I=I_{L / K}$ denote the group of fractional ideals of $K$ whose prime decomposition contains no prime ideals that ramify in $L$ and let $P=\left\{(\alpha) \in I: \alpha \in K^{*}, \alpha \succ 0\right\}$. Following Hecke [9] let $\left(\lambda_{1}, \lambda_{2}, \ldots, \lambda_{n-1}\right)$ be a basis for the torsion-free characters on $P$ that satisfy $\lambda_{i}(\varepsilon)=1,1 \leq i \leq n-1$, for all units $\varepsilon \succ 0$ in $\mathcal{O}_{K}$, the ring of integers of $K$. Fixing an extension of each $\lambda_{i}$ to a character on $I$ then $\lambda_{i}(\mathfrak{a}), 1 \leq i \leq n-1$ are defined for all ideals $\mathfrak{a}$ of $K$ that do not ramify in $L$. So for such ideals we can define $\psi(\mathfrak{a})=\left(\psi_{i}(\mathfrak{a})\right) \in \mathbb{R}^{n-1} / \mathbb{Z}^{n-1}=\mathbb{T}^{n-1}$ by $\lambda_{j}(\mathfrak{a})=e^{2 \pi i \psi_{j}(\mathfrak{a})}$. Then the small region of $K$ referred to above is

$$
\begin{aligned}
\mathcal{S}\left(x, \psi_{0}, \ell\right)=\{\mathfrak{a} \in I: x(1-\ell) \leq N \mathfrak{a} \leq x(1+\ell), & \\
& \left.\left|\psi_{j}(\mathfrak{a})-\psi_{0}\right|_{\mathbb{T}} \leq \ell, 1 \leq j \leq n-1\right\}
\end{aligned}
$$

for $0<\ell<\frac{1}{2}$, and $\psi_{0} \in \mathbb{T}^{n-1}$, with the notation that, for any $\alpha \in \mathbb{R}$ we set $|\alpha|_{\mathbb{T}}=\beta$ where $\beta$ is the unique real satisfying $-\frac{1}{2}<\beta \leq \frac{1}{2}, \beta \equiv \alpha(\bmod 1)$. In [1] and Theorem 2 of [4] we showed that we can count the number of prime ideals in $\mathcal{S}\left(x, \psi_{0}, \ell\right)$, when $L=K$, as long as $\ell>x^{-5 / 12 n_{K}+\varepsilon}$. The method of proof is by standard methods of contour integration and Dirichlet series. If we were only interested in enforcing the one condition $x(1-\ell) \leq N \mathfrak{a} \leq x(1+\ell)$, then the Dirichlet series considered would be the Dedekind zeta function in $K$ or, if there are congruent conditions on the prime ideals, the Hecke-Landau $L$-functions with characters of finite 
order. See, for example, Sokolovskii [25] for such results. To deal with the restrictions on the $\psi_{j}(\mathfrak{a})$ it is necessary to use Fourier series which leads to terms $\prod_{j=1}^{n-1} e^{2 \pi i m_{j} \psi_{j}(\mathfrak{a})}=\prod_{j=1}^{n-1} \lambda_{j}^{m_{j}}(\mathfrak{a})$, denoted by $\lambda^{\overrightarrow{\mathbf{m}}}(\mathfrak{a}), \overrightarrow{\mathbf{m}}=$ $\left(m_{1}, m_{2}, \ldots, m_{n-1}\right) \in \mathbb{N}_{0}^{n-1}$ where $\mathbb{N}_{0}=\mathbb{N} \cup\{0\}$. These are examples of groessencharaktere, characters of infinite order. So now we need to consider Hecke $L$-functions with groessencharaktere. These go back to Hecke [9] but see also Kubilius [14], [15] or Fogels [8] for examples of uses of such Dirichlet series.

In this paper we consider $\theta$, a complex-valued multiplicative function of ideals of $I$ such that, if the Frobenius classes in $G=\operatorname{Gal}(L / K)$ of different prime ideals $\mathfrak{p}$ and $\mathfrak{q}$ of $I$ are equal, that is $[(L / K) / \mathfrak{p}]=[(L / K) / \mathfrak{q}]$, then $\theta\left(\mathfrak{p}^{n}\right)=\theta\left(\mathfrak{q}^{n}\right)$ for all $n \geq 1$. Following [21] we will say that $\theta$ is Frobenius with respect to $G$. Similarly let $\Theta$ be a Frobenius multiplicative function with respect to $G$ but with values in some finite multiplicative monoid $\Gamma=$ $\left\{\gamma_{1}, \gamma_{2}, \ldots, \gamma_{t}\right\}$, say. Then, for each $1 \leq i \leq t$, we can define

$$
\omega_{i}(\mathfrak{a})=\#\left\{\mathfrak{p}^{n} \| \mathfrak{a}, \Theta\left(\mathfrak{p}^{n}\right)=\gamma_{i}\right\} \quad \text { and } \quad \Omega_{i}(\mathfrak{a})=\sum_{\substack{\mathfrak{p}^{n} \| \mathfrak{a} \\ \Theta\left(\mathfrak{p}^{n}\right)=\gamma_{i}}} n
$$

We will consider vector-valued functions $\mathbf{f}(\mathfrak{a})=\left(f_{i}(\mathfrak{a})\right)_{1 \leq i \leq t}$ where, for each $1 \leq i \leq t, f_{i}(\mathfrak{a})$ is either $\omega_{i}(\mathfrak{a}), \Omega_{i}(\mathfrak{a})$ or $\Omega_{i}(\mathfrak{a})-\omega_{i}(\mathfrak{a})$. For $\mathbf{z}=$ $\left(z_{1}, \ldots, z_{t}\right) \in \mathbb{C}^{t}$ define

$$
\mathbf{z}^{\mathbf{f}(\mathfrak{a})}=\prod_{\substack{i=1 \\ f_{i}(\mathfrak{a}) \neq 0}}^{t} z_{i}^{f_{i}(\mathfrak{a})}
$$

In the first result of this paper we evaluate $\sum_{\mathfrak{a} \in \mathcal{S}\left(x, \psi_{0}, \ell\right)} \theta(\mathfrak{a}) \mathbf{z}^{\mathbf{f}(\mathfrak{a})}$. The Huxley-Hooley method reduces this sum to an integral of a Dirichlet series, see equations (6) and (7) below. This integral is of a type seen in proofs of Selberg-Delange type results, as seen in [26], Chapter II.5 or [29]. As a corollary of this result we are able to count the number of ideals $\mathfrak{a} \in \mathcal{S}\left(x, \psi_{0}, \ell\right)$ that are the relative norms of ideals from some given number field extension of $K$. The original problem in [22] of counting squares and sums of two 
squares, which spurred on the development of the Hooley-Huxley method, is one of counting rational integers that are the norms of ideals in $\mathbb{Q}(i)$. The main motivation of this paper is, though, the counting of irreducible integers $\alpha \in K$ satisfying $(\alpha) \in \mathcal{S}(x, \psi, \ell)$. This is Theorem 7 below.

1.1 First Result Given a conjugacy class, $C$ in $G$, we know that $\Theta(\mathfrak{p})$, and thus $\mathbf{f}(\mathfrak{p})$, is constant for all $\mathfrak{p}$ with $[(L / K) / \mathfrak{p}]=C$ and so we can write $z_{C}$ for the value of $\mathbf{z}^{\mathbf{f}(\mathfrak{p})}$ for any such $\mathfrak{p}$. We can now define $\alpha(\mathbf{z})=$ $\sum_{C}|C| \theta(C) z_{C} /|G|$ with the obvious meaning for $\theta(C)$. We now have all the notation necessary to state our first result

THEOREM 1 Let $A>0$ be given and define $A_{i}, 1 \leq i \leq t$ by $A_{i}=A$ if $f_{i}=\omega_{i}$ and $A_{i}=q_{0}^{1 / 4}$, where $q_{0}$ is the smallest norm of the prime ideals of $K$, if $f_{i}=\Omega_{i}$ or $\Omega_{i}-\omega_{i}$. Assume that $\left|z_{i}\right|<A_{i}$ for all $1 \leq i \leq t$.

Let $\mathfrak{p} \in I$. Assume that if $\Theta\left(\mathfrak{p}^{n}\right)=\gamma_{i}$ and if $f_{i}=\omega_{i}$ then $\theta\left(\mathfrak{p}^{n}\right) \ll c^{n}$ for some $c<q_{0}^{1 / 2}$ while if $f_{i}=\Omega_{i}$ or $\Omega_{i}-\omega_{i}$ then $\theta\left(\mathfrak{p}^{n}\right) \ll c^{n}$ for some $c<q_{0}^{1 / 4}$.

Let $M=\sup |\alpha(\mathbf{z})|$, where the supremum is over allowable $\mathbf{z}$. Let $\varepsilon>0$ be given and assume that $\ell$ satisfies

$$
\frac{1}{2}>\ell> \begin{cases}x^{-5(1-\varepsilon) / 12 n_{K}} & \text { if } L / K \text { abelian } \\ x^{-3(1-\varepsilon) / 2\left(n_{L}+3 n_{K}\right)} & \text { otherwise. }\end{cases}
$$

Then for all $\psi_{0} \in \mathbb{T}^{n_{K}-1}$ we have

$$
\begin{aligned}
\sum_{\substack{\mathfrak{a} \in \mathcal{S}\left(x, \psi_{0}, \ell\right) \\
\mathfrak{a} \text { integral }}} \theta(\mathfrak{a}) \mathbf{z}^{\mathbf{f}(\mathfrak{a})}= & \frac{x(2 \ell)^{n_{K}}}{(\log x)^{1-\alpha(\mathbf{z})}} \sum_{j=0}^{J} \frac{c_{j}(\mathbf{z})}{(\log x)^{j}} \\
& +x \ell^{n_{K}} E_{J}(\mathbf{z})+O\left(x \ell^{n_{K}} \exp (-R(x))\right),
\end{aligned}
$$

for some coefficients $c_{j}(\mathbf{z})$, with $R(x)=\kappa_{1}(\log x)^{1 / 3}(\log \log x)^{-1 / 3}$. Here $E_{J}(\mathbf{z})=0$ if $\alpha(\mathbf{z})$ is an integer while otherwise

$$
E_{J}(\mathbf{z}) \ll_{M} \frac{1}{(\log x)^{1-\operatorname{Re} \alpha(\mathbf{z})}}\left\{\left(\frac{c_{1} J+1}{\log x}\right)^{J+1}+\ell\left(c_{0} \log x\right)^{M}\right\}
$$


subject to $J+M+1 \leq c_{0} \log x$. Here, $\kappa_{1}$ and $c_{1}$ are constants that depend on $M$ as well as $K$ and $L$ while $c_{0}$ depends only on $L$ and $K$.

From the proof we see that if $J \ll M$ then the last term in (3) does not occur. If $J=J(x)=\kappa_{2}(\log x)^{1 / 3}(\log \log x)^{-4 / 3}$ and $\ell \leq \exp (-R(x))$ then $E_{J} \ll x \ell^{n_{K}} \exp \left(-R(x)\right.$ ) (with the convention that $\kappa_{1}$ need not be the same at each occurrence). Note that $M \ll_{k} A$.

PROOF The sum on the left hand side of (2) is a particular example of the quantity $A\left(x, \psi_{0}, \ell\right)$ defined on p.251 of [5]. This quantity is seen, in equation (21) of [5], to be related to the Dirichlet series

$$
F(s, \overrightarrow{\mathbf{m}}, \mathbf{z})=\sum_{\substack{\mathfrak{a} \in I \\ \mathfrak{a} \text { integral }}} \frac{\theta(\mathfrak{a}) \mathbf{z}^{\mathbf{f}(\mathfrak{a})} \lambda^{\overrightarrow{\mathbf{m}}}(\mathfrak{a})}{N \mathfrak{a}^{s}} .
$$

In examining for what $s$ this series converges we consider the Euler product $\prod_{\mathfrak{p} \in I}\left(1+U_{\mathfrak{p}}(s, \overrightarrow{\mathbf{m}}, \mathbf{z})\right)$, where

$$
U_{\mathfrak{p}}(s, \overrightarrow{\mathbf{m}}, \mathbf{z})=\sum_{n \geq 1} \frac{\theta\left(\mathfrak{p}^{n}\right) \mathbf{z}^{\mathbf{f}\left(\mathfrak{p}^{n}\right)} \lambda^{\overrightarrow{\mathbf{m}}}\left(\mathfrak{p}^{n}\right)}{N \mathfrak{p}^{n s}} .
$$

The analytic properties of Euler products are dictated by the $n=1$ terms in $U_{\mathfrak{p}}(s, \overrightarrow{\mathbf{m}}, \mathbf{z})$. Given a prime $\mathfrak{p} \in I$, we can construct a characteristic function for the conjugacy class that contains $\mathfrak{p}$ by using characters on $G$. For each irreducible character $\phi$ on $G$, let $\phi_{K}\left(\mathfrak{p}^{n}\right)=\phi\left(\left[(L / K) / \mathfrak{p}^{n}\right]\right)$ for primes unramified in $L$. For each conjugacy class $C$ choose an element $g \in C$, let $H=\langle g\rangle$ be the cyclic group generated by $g, E$ the fixed field of $H$ and let $\chi$ denote irreducible characters of $H$. Then it is known, see for example p.422 of [16], that for $\mathfrak{p} \in I$

$$
\frac{\theta(\mathfrak{p}) \mathbf{z}^{\mathbf{f}(\mathfrak{p})} \lambda^{\overrightarrow{\mathbf{m}}}(\mathfrak{p})}{N \mathfrak{p}^{s}}=\sum_{C} z_{C} \theta(C) \frac{|C|}{|G|} \sum_{\phi} \bar{\phi}(g) \phi_{K}(\mathfrak{p}) \frac{\lambda^{\overrightarrow{\mathbf{m}}}(\mathfrak{p})}{N \mathfrak{p}^{s}} .
$$

Also, from p. 430 of [16] we have

$$
\sum_{\phi} \bar{\phi}(g) \phi=\sum_{\chi} \bar{\chi}(g) \chi^{*}
$$


where $\chi^{*}$ is the character on $G$ induced from $\chi$ on $H$. Thus

$$
\frac{\theta(\mathfrak{p}) \mathbf{z}^{\mathbf{f}(\mathfrak{p})} \lambda^{\overrightarrow{\mathbf{m}}}(\mathfrak{p})}{N \mathfrak{p}^{s}}=\sum_{C} z_{C} \theta(C) \frac{|C|}{|G|} \sum_{\chi} \bar{\chi}(g) \frac{\chi_{K}^{*}(\mathfrak{p}) \lambda^{\overrightarrow{\mathbf{m}}}(\mathfrak{p})}{N \mathfrak{p}^{s}} .
$$

Now, for $\mathfrak{p} \in I$, define

$$
V_{\mathfrak{p}}(s, \overrightarrow{\mathbf{m}}, \mathbf{z})=\sum_{C} z_{C} \theta(C) \frac{|C|}{|G|} \sum_{\chi} \bar{\chi}(g) \sum_{r \geq 1} \frac{\chi_{K}^{*}\left(\mathfrak{p}^{r}\right) \lambda^{\overrightarrow{\mathbf{m}}}\left(\mathfrak{p}^{r}\right)}{r N \mathfrak{p}^{r s}} .
$$

Thus the $r=1$ term for $V_{\mathfrak{p}}(s, \overrightarrow{\mathbf{m}}, \mathbf{z})$ equals the $n=1$ term for $U_{\mathfrak{p}}(s, \overrightarrow{\mathbf{m}}, \mathbf{z})$. This is important when we apply a result due to Delange [6] rewritten in [5] as

LEMMA 1 Assume that $\left\{U_{\mathfrak{p}}(s, \mathbf{z})\right\}_{\mathfrak{p}}$ and $\left\{V_{\mathfrak{p}}(s, \mathbf{z})\right\}_{\mathfrak{p}}$ are sequences of complex valued functions defined on $\mathbb{C} \times \mathbb{C}^{N}$. Assume that on some domain $\mathcal{D} \subseteq$ $\mathbb{C} \times \mathbb{C}^{N}$ there exist, for all $\mathfrak{p}$, positive constants $U_{\mathfrak{p}}$ and $V_{\mathfrak{p}}$ satisfying

$$
\left|U_{\mathfrak{p}}(s, \mathbf{z})\right| \leq U_{\mathfrak{p}}, \quad\left|U_{\mathfrak{p}}(s, \mathbf{z})-V_{\mathfrak{p}}(s, \mathbf{z})\right| \leq V_{\mathfrak{p}}
$$

along with

$$
\sum_{\mathfrak{p}} U_{\mathfrak{p}}^{2}<\infty \quad \text { and } \quad \sum_{\mathfrak{p}} V_{\mathfrak{p}}<\infty .
$$

Then the infinite product

$$
\prod_{\mathfrak{p}}\left(1+U_{\mathfrak{p}}(s, \mathbf{z})\right) \exp \left(-V_{\mathfrak{p}}(s, \mathbf{z})\right)
$$

is absolutely and uniformly convergent on $\mathcal{D}$ and bounded on $\mathcal{D}$.

By the conditions of the theorem we have, whatever the choice of $\mathbf{f}$, that $\left|\theta\left(\mathfrak{p}^{n}\right) \mathbf{z}^{\mathbf{f}\left(\mathfrak{p}^{n}\right)}\right| \ll c^{n}$ with some $c<q_{0}^{1 / 2}$ where $q_{0}$ is the smallest norm of the prime ideals of $K$. Hence we can show that, as in section 2 of [5], given $\sigma_{1}>\frac{1}{2}$ then, for $\operatorname{Re} s \geq \sigma_{1}$, we have

$$
\left|U_{\mathfrak{p}}(s, \overrightarrow{\mathbf{m}}, \mathbf{z})\right| \leq \frac{c_{2}}{N \mathfrak{p}^{\sigma_{1}}}+c_{3}\left(\frac{c}{N \mathfrak{p}^{\sigma_{1}}}\right)^{2},
$$

with constants $c_{2}=c_{2}(A, \theta), c_{3}=c_{3}\left(A, \theta, c, \sigma_{1}\right)$. It is then easy to see that both conditions of the lemma hold and that we can conclude that

$$
F_{0}(s, \overrightarrow{\mathbf{m}}, \mathbf{z}):=\prod_{\mathfrak{p} \in I}\left(1+U_{\mathfrak{p}}(s, \overrightarrow{\mathbf{m}}, \mathbf{z})\right) \exp \left(-V_{\mathfrak{p}}(s, \overrightarrow{\mathbf{m}}, \mathbf{z})\right)
$$


converges absolutely and uniformly for $\operatorname{Re} s \geq \sigma_{1}$, all $\overrightarrow{\mathbf{m}}$ and all $\mathbf{z}$ satisfying the conditions of Theorem 1. Now

$\prod_{\mathfrak{p} \in I} \exp \left(V_{\mathfrak{p}}(s, \overrightarrow{\mathbf{m}}, \mathbf{z})\right)=\exp \left(\sum_{C} z_{C} \theta(C) \frac{|C|}{|G|} \sum_{\chi} \bar{\chi}(g) \sum_{\mathfrak{p} \in I} \sum_{r \geq 1} \frac{\chi_{K}^{*}\left(\mathfrak{p}^{r}\right) \lambda^{\overrightarrow{\mathbf{m}}}\left(\mathfrak{p}^{r}\right)}{r N_{K} \mathfrak{p}^{r s}}\right)$.

From the study of the contribution to $L$-series of induced characters, as described in Chapter XII, Section 3, of [18], we see that the inner series over $r$ equals

$$
\sum_{\mathfrak{q} \mid \mathfrak{p}} \sum_{n \geq 1} \frac{\chi_{E}\left(\mathfrak{q}^{n}\right) \lambda^{\overrightarrow{\mathbf{m}}}\left(N_{E / K} \mathfrak{q}^{n}\right)}{n N_{E} \mathfrak{q}^{n s}}
$$

where the outer sum is over prime ideals $\mathfrak{q}$ of $E$ lying over $\mathfrak{p}$, and where $\chi_{E}$ is now considered to be a character on the narrow ideal classes mod $\mathfrak{f}$ of $E$ for some conductor $\mathfrak{f}$. Hence the inner double sum differs by only a finite number of primes from $\log L\left(s, \chi_{E} \lambda_{E / K}^{\overrightarrow{\mathbf{m}}}\right)$ where $L\left(s, \chi_{E} \lambda_{E / K}^{\overrightarrow{\mathbf{m}}}\right)$ is defined by

$$
\sum_{(\mathfrak{a}, \mathfrak{f})=1} \frac{\chi_{E}(\mathfrak{a}) \lambda^{\overrightarrow{\mathbf{m}}}\left(N_{E / K} \mathfrak{a}\right)}{N_{E} \mathfrak{a}^{s}}
$$

for $\operatorname{Re} s>1$. This finite number of primes can be absorbed into $F_{0}(s, \overrightarrow{\mathbf{m}}, \mathbf{z})$. Hence

$$
F(s, \overrightarrow{\mathbf{m}}, \mathbf{z})=F_{1}(s, \overrightarrow{\mathbf{m}}, \mathbf{z}) \prod_{C} \prod_{\chi} L\left(s, \chi_{E} \lambda_{E / K}^{\overrightarrow{\mathbf{m}}}\right)^{\alpha(C, \chi, \mathbf{z})},
$$

where $F_{1}(s, \overrightarrow{\mathbf{m}}, \mathbf{z})$ satisfies the same properties as $F_{0}(s, \overrightarrow{\mathbf{m}}, \mathbf{z})$ and $\alpha(C, \chi, \mathbf{z})=$ $|C| \theta(C) \bar{\chi}(g) z_{C} /|G|$. The $L\left(s, \chi_{E} \lambda_{E / K}^{\overrightarrow{\mathbf{m}}}\right)$ are $L$-functions in $E$ with groessencharaktere from $K$.

The Hooley-Huxley method of [5] firstly deals with the weighted sum

$$
\sum_{\mathfrak{a}} \theta(\mathfrak{a}) \mathbf{z}^{\mathbf{f}(\mathfrak{a})} \theta_{x, \psi}(\mathfrak{a}) \approx \frac{1}{2 \pi i} \sum_{\|\overrightarrow{\mathbf{m}}\|<W} a_{\overrightarrow{\mathbf{m}}} e^{-2 \pi i \overrightarrow{\mathbf{m}} \cdot \psi_{0}} \int_{c-i W}^{c+i W} \widehat{g}(s) F(s, \overrightarrow{\mathbf{m}}, \mathbf{z}) d s
$$

where $\|\overrightarrow{\mathbf{m}}\|=\max _{1 \leq i \leq t}\left|m_{i}\right|$. The weight $\theta_{x, \psi}$ is a product of continuous functions approximating the characteristic functions of either the interval $[x(1-\ell), x(1+\ell)]$ for $N \mathfrak{a}$ or intervals $(-\ell, \ell)$ for each of the $\psi_{i}(\mathfrak{a})$. The 
approximations differ from the characteristic functions on intervals of length $\ll x \Delta$ or $\ll \Delta$, respectively. The $\widehat{g}(s)$ and $a_{\overrightarrow{\mathbf{m}}}$ arise in (5) as either the Mellin transforms or Fourier coefficients of the approximations, and satisfy $a_{\overrightarrow{\mathbf{m}}} \ll a_{\overrightarrow{\mathbf{0}}}=(2 \ell+O(\Delta))^{n-1}$ for all $\overrightarrow{\mathbf{m}}$ and $\widehat{g}(\sigma+i t) \ll \ell x^{\sigma}$ for all $t$. The truncation $W$ can be taken as $\left(\log ^{3} x\right) / \Delta$ and the notation $\approx$ in $(5)$ means that the two sides differ by an arbitrary small function of $x$.

The lines of integration in (5) are moved to the left of Re $s=1$, though when $\overrightarrow{\mathbf{m}}=\overrightarrow{\mathbf{0}}$ we have to retain a loop about $s=1$. The final position of the lines depend on the position of the zeros of the $L$-functions in (4) and on the growth of these functions. Their growth on vertical lines is measured in terms of $V(\overrightarrow{\mathbf{m}}, t)^{n_{E}}$ where $n_{E}=\operatorname{deg} E / \mathbb{Q}$, and $V(\overrightarrow{\mathbf{m}}, t)=$ $\left\{1+t^{2}+\sum m_{i}^{2}\right\}^{1 / 2}$ while in [5] growth of the $L$-functions that occurred there was measured in terms of $V(\overrightarrow{\mathbf{m}}, t)^{n_{K}}$, which has a smaller exponent. Yet, the arguments can be followed through Lemma 14 and equations (32) and (34) of [5] to see that the change in exponent can only alter the constant, $\kappa_{1}$, in our definition of $R(x)$. We have the same quality of zero-free region for the collection of $L$-functions in $F(s, \overrightarrow{\mathbf{m}}, \mathbf{z})$ as we had in [5], the only difference being the dependency of a constant on the fields $E$ that arise and this only effects the implicit constant in our final result (2).

We now apply zero-density results, that is, bounds for

$$
N_{E / K}(\sigma, W)=\sum_{\|\overrightarrow{\mathbf{m}}\|<W} \sum_{\chi} \sum_{\substack{\left|\gamma_{\overrightarrow{\mathbf{m}} \chi}\right|<W \\ \beta_{\overrightarrow{\mathbf{m}} \chi} \geq \sigma}} 1
$$

where $\rho_{\overrightarrow{\mathbf{m}} \chi}=\beta_{\overrightarrow{\mathbf{m}} \chi}+i \gamma_{\overrightarrow{\mathbf{m}} \chi}$ satisfies $L\left(\rho_{\overrightarrow{\mathbf{m}} \chi}, \chi_{E} \lambda_{E / K}^{\overrightarrow{\mathbf{m}}}\right)=0$. From Theorem 5 of [4] we have that, for any $\varepsilon>0$,

$$
N_{E / K}(\sigma, W) \ll \begin{cases}W^{\left(12 n_{K} / 5+\varepsilon\right)(1-\sigma)}(\log W)^{c_{4}} & \text { if } E / K \text { is abelian, } \\ W^{\left(2\left(n_{E}+3 n_{K}\right) / 3\right)(1-\sigma)}(\log W)^{c_{5}} & \text { otherwise }\end{cases}
$$

uniformly for $1 / 2 \leq \sigma \leq 1$, where $c_{4}$ depends on $\varepsilon$. There are stronger results in [4] for when $n_{E} \leq 5 n_{K}$, but to get any advantage from these we 
need to have $n_{E} \leq 5 n_{K}$ for all $E \subseteq L$, in which case $n_{L} \leq 5 n_{K}$. But then $L / K$ must necessarily be abelian, given it is Galois, as must be then every $E / K$, and we can use the first of the bounds for $N_{E / K}(\sigma, W)$ on each $E$, giving a result depending only on $K$. So the additional results in [4] do not improve the bounds in (1).

Given a zero density bound of the form $W^{B(1-\sigma)} \log ^{c} W$, the methods of [5] can be used to estimate the contribution of the new lines of integration, as long as $\ell>x^{-(1-\varepsilon) / B}$. The weights $\theta_{x, \psi}$ can then be "stripped" from (5) and we can prove the following version of Theorem 1 of [5]: Under the conditions of Theorem 1 we have

$$
\sum_{\substack{\mathfrak{a} \in \mathcal{S}\left(x, \psi_{0}, \ell\right) \\ \mathfrak{a} \text { integral }}} \theta(\mathfrak{a}) \mathbf{z}^{\mathbf{f}(\mathfrak{a})}-I(x, \ell) \ll x \ell^{n_{K}} \exp (-R(x))
$$

where

$$
I(x, \ell)=\frac{(2 \ell)^{n_{K}-1}}{2 \pi i} \int_{x(1-\ell)}^{x(1+\ell)} \int_{\mathcal{C}_{0}} y^{s-1} F(s, \overrightarrow{\mathbf{0}}, \mathbf{z}) d s d y .
$$

Here $\mathcal{C}_{0}=\left\{s \in \mathbb{C}:|s-1|=c_{0}, s \neq 1-c_{0}\right\}$ is traversed in the anti-clockwise direction, and $c_{0}$ is chosen so that $F(s, \overrightarrow{\mathbf{0}}, \mathbf{z})$ has no singularities on the boundary or in the interior of the circle of radius $3 c_{0}$ with centre 1 except for $s=1$. The evaluation of the integral over $\mathcal{C}_{0}$ is a standard calculation in proofs of Selberg-Delange type formula; see, for example Chapter II.5 of [26] and, in particular, pages 189 and 190 of that book. From (4) we have, for $|s-1| \leq 3 c_{0}$, that

$$
F(s, \overrightarrow{\mathbf{0}}, \mathbf{z})=\left(\frac{1}{s-1}\right)^{\alpha(\mathbf{z})} H(s, \mathbf{z}),
$$

where $\alpha(\mathbf{z})=\sum_{C} \alpha(C, 1, \mathbf{z})$ and $H(s, \mathbf{z})$ is analytic in $|s-1| \leq 3 c_{0}$ for those $\mathbf{z}$ of Theorem 1. If $\alpha(\mathbf{z})$ is a non-positive integer, then there is no pole in the integrand in (5) and so no main term in (2). If $\alpha(\mathbf{z})$ is a positive integer, then we can move the line of integration over the pole and we get a 
contribution, the number of terms of which is independent of $x$. Otherwise we expand $H(s, \mathbf{z})$ about $s=1$ as

$$
\begin{aligned}
H(s, \mathbf{z}) & =\sum_{j=0}^{\infty} h_{j}(\mathbf{z})(s-1)^{j} \\
& =\sum_{j=0}^{J} h_{j}(\mathbf{z})(s-1)^{j}+O\left(\left(\frac{|s-1|}{2 c_{0}}\right)^{J+1}\right),
\end{aligned}
$$

valid for $s \in \mathcal{C}_{0}$, all $\mathbf{z}$ of Theorem 1 and all $J \geq 1$. The error here arises from the bound $h_{j}(\mathbf{z}) \ll\left(2 c_{0}\right)^{-j}$ for all $j \geq 1$, valid for all $\mathbf{z}$ of Theorem 1 . The integrals over $\mathcal{C}_{0}$ of the $J+1$ terms in the sum are truncated Hankel integrals. As in the derivation of equation (24) on p.190 of [26] we can deduce that

$$
\begin{aligned}
& \frac{1}{2 \pi i} \int_{\mathcal{C}_{0}} y^{s-1} F(s, \overrightarrow{\mathbf{0}}, \mathbf{z}) d s \\
& \quad=\frac{1}{(\log y)^{1-\alpha(\mathbf{z})}}\left(\sum_{j=0}^{J} \frac{h_{j}(\mathbf{z})}{\Gamma(\alpha(\mathbf{z})-j)(\log y)^{j}}+O\left(\left(\frac{c_{1} J+1}{\log y}\right)^{J+1}\right)\right)
\end{aligned}
$$

There is no condition on $J$ in this result. The error here, the first in $E_{J}$, also dominates the contribution from the error in (8). For the integral over $y$ in (7) we use

$$
\int_{x(1-\ell)}^{x(1+\ell)} \frac{d y}{(\log y)^{\gamma}}-\frac{2 \ell x}{(\log x)^{\gamma}} \ll \frac{|\gamma| x \ell^{2}}{(\log x)^{\operatorname{Re} \gamma+1}} .
$$


Using this on the terms in the sum in (9) gives an error

$$
\begin{aligned}
& \ll \quad x \ell^{2} \sum_{j=0}^{J} \frac{|j+1-\alpha(\mathbf{z})|}{\left(2 c_{0}\right)^{j}|\Gamma(\alpha(\mathbf{z})-j)|(\log x)^{j+2-\operatorname{Re} \alpha(\mathbf{z})}} \\
& \ll \quad M \frac{x \ell^{2}}{(\log x)^{1-\operatorname{Re} \alpha(\mathbf{z})}}\left(1+\frac{1}{|\Gamma(\alpha(\mathbf{z})-2 M)|}\right. \\
& \left.\quad \times \sum_{j=2 M}^{J} \frac{|j+1-\alpha(\mathbf{z})||j-\alpha(\mathbf{z})| \ldots|2 M+1-\alpha(\mathbf{z})|}{\left(2 c_{0} \log x\right)^{j+1}}\right) \\
& \ll \quad M \frac{x \ell^{2}}{(\log x)^{1-\operatorname{Re} \alpha(\mathbf{z})} \sum_{j=0}^{J} \frac{(j+M+1) !}{M !\left(2 c_{0} \log x\right)^{j+1}}} \\
& \ll \quad M \frac{x \ell^{2}\left(2 c_{0} \log x\right)^{M}}{(\log x)^{1-\operatorname{Re} \alpha(\mathbf{z})} \sum_{j=0}^{J}}\left(\frac{J+M+1}{2 c_{0} \log x}\right)^{j+M+1}
\end{aligned}
$$

which leads to the second term in $E_{J}$ when $J+M+1<c_{0} \log x$.

The leading coefficient, $c_{0}(\mathbf{z})$, of $(2)$ equals $h_{0}(\mathbf{z}) / \Gamma(\alpha(\mathbf{z}))=H(1, \mathbf{z}) / \Gamma(\alpha(\mathbf{z}))$. In applications we will often write $c_{0}(\mathbf{z})=\alpha(\mathbf{z}) U(\mathbf{z})$. Importantly, if $\alpha(\mathbf{z})=$ 0 then $U(\mathbf{z}) \neq 0$ though $c(\mathbf{z})=0$.

Note that

$$
H(s, \mathbf{z})=(s-1)^{\alpha(\mathbf{z})} F(s, \overrightarrow{\mathbf{0}}, \mathbf{z})=\left(\frac{\rho_{K}}{\zeta_{K}(s)}\right)^{\alpha(\mathbf{z})} F(s, \overrightarrow{\mathbf{0}}, \mathbf{z}) h(s)^{\alpha(\mathbf{z})},
$$

where $\rho_{K}$ is the residue of the Dedekind zeta-function of $K \zeta_{K}(s)$ at $s=1$, and $h(s)$ is a function analytic at $s=1$ with $h(1)=1$. Hence $H(1, \mathbf{z})=\rho_{K}^{\alpha(\mathbf{z})} \prod_{\mathfrak{p} \notin I}\left(1-\frac{1}{N \mathfrak{p}}\right)^{\alpha(\mathbf{z})} \prod_{\mathfrak{p} \in I}\left(1+\sum_{n \geq 1} \frac{\theta\left(\mathfrak{p}^{n}\right) \mathbf{z}^{\mathbf{f}\left(\mathfrak{p}^{n}\right)}}{N \mathfrak{p}^{n}}\right)\left(1-\frac{1}{N \mathfrak{p}}\right)^{\alpha(\mathbf{z})}$. NOTES (i) If $\ell=1 / 2$, the bound (3) on the error $x \ell^{n_{K}} E_{J}(x)$ dominates the main terms of (2). We should expect this since the coefficients $c_{j}(\mathbf{z})$ constructed in the proof are not what appear when $\ell=1 / 2$. The proof when $\ell=1 / 2$ differs from above in that we require an expansion for $s^{-1} H(s, \mathbf{z})$ about $s=1$ in place of (8). This leads to a result of type (2) with $c_{j}(\mathbf{z})$ replaced by $d_{j}(\mathbf{z})$ defined by $d_{0}(\mathbf{z})=c_{0}(\mathbf{z})$ and $d_{j}(\mathbf{z})-c_{j}(\mathbf{z})=(j-\alpha(\mathbf{z})) d_{j-1}(\mathbf{z})$ for all $j \geq 1$. 
(ii) If $f_{i}=\Omega_{i}-\omega_{i}$ for all $1 \leq i \leq t$, then $z_{C}=1$ for all conjugacy classes. If, further, $\theta \equiv 1$, then $\alpha(\mathbf{z})=\sum_{C}|C| /|G|=1$ for all $\mathbf{z}$. In this case there is only the $j=0$ term in the sum on the right hand side of (2). With further work we can extract terms of lower order. By the methods of the above proof we can show that, in the present case,

$$
F(s, \overrightarrow{\mathbf{m}}, \mathbf{z})=F_{2}(s, \overrightarrow{\mathbf{m}}, \mathbf{z}) L\left(s, \lambda^{\overrightarrow{\mathbf{m}}}\right) \prod_{C} \prod_{\chi} L\left(2 s, \chi_{E} \lambda_{E / K}^{2 \overrightarrow{\mathbf{m}}}\right)^{\beta(C, \chi, \mathbf{z})},
$$

where $\beta(C, \chi, \mathbf{z})=|C|\left(z_{C}^{*}-1\right) \bar{\chi}(g) /|G|$. Here $z_{C}^{*}=\prod_{i=1}^{t} z_{i}^{\left(\Omega_{i}-\omega_{i}\right)\left(\mathfrak{p}^{2}\right)}$, for any $\mathfrak{p}:\left[\frac{L / K}{\mathfrak{p}}\right] \in C$, and so $z_{C}^{*}=z_{j}$ where $j$ is given by $\Theta\left(\mathfrak{p}^{2}\right)=\gamma_{j}$ for such $\mathfrak{p}$. The factor $F_{2}(s, \overrightarrow{\mathbf{m}}, \mathbf{z})$ converges absolutely and uniformly for $\operatorname{Re} s \geq \sigma_{2}$ for any $\sigma_{2}>1 / 3$, and is bounded in any such half plane for all $\overrightarrow{\mathbf{m}}$ and for all $\mathbf{z}$ satisfying the conditions of Theorem 1 .

If we had factorized $\mathbf{z}^{f(\mathfrak{a})}=\left(1 * g_{\mathbf{z}}\right)(\mathfrak{a})$, then the Dirichlet series for $g_{\mathbf{z}}(\mathfrak{a})$ would have the factorization (10) but without the $L\left(s, \lambda^{\overrightarrow{\mathbf{m}}}\right)$ term. The ideas of Theorem 1 would then give a local result for $g_{\mathbf{z}}(\mathfrak{a})$, with main term $(2 \ell)^{n_{K}} x^{1 / 2} c(\mathbf{z}) /(\log x)^{\beta(\mathbf{z})-2}$ where $\beta(\mathbf{z})=\sum_{C}|C| z_{C}^{*} /|G|$, subject to either $\ell>x^{-5(1-\varepsilon) / 24 n_{K}}$ or $\ell>x^{-3(1-\varepsilon) / 4\left(n_{L}+3 n_{K}\right)}$. But it would then be difficult to recover a similar result for $\mathbf{z}^{f(\mathfrak{a})}$. The Dirichlet-Hyperbola method as used by Delange [7] and $\mathrm{Wu}$ [28] in similar problems for rational integers fails because of the large errors encountered when counting the number of ideals with norms in given intervals.

Instead we consider (10) directly and apply the methods of Wolke [27]. So now the lines of integration in (5) are moved back to $\operatorname{Re} s=\frac{1}{2}-$ $c_{6} L^{-2 / 3} L_{2}^{-1 / 3}=\frac{1}{2}-\eta$, say, where $L=\log W$ and $L_{2}=\log L$. Apart from the value of $c_{6}$, this is the edge of the zero-free region for the $L\left(2 s, \chi_{E} \lambda_{E / K}^{2 \overrightarrow{\mathbf{m}}}\right)$ factors in (10), when $|t| \leq W$ and $\|\overrightarrow{\mathbf{m}}\|<W$ (see [2]). Because of a possible singularity we have to keep a loop, $\mathcal{B}$, around $s=1 / 2$ when $\overrightarrow{\mathbf{m}}=\overrightarrow{\mathbf{0}}$. If we try to move the vertical line of integration further to the left with the intention of using the ideas of the Hooley-Huxley method it becomes difficult to 
estimate the $L\left(s, \lambda^{\overrightarrow{\mathbf{m}}}\right)$ factor in (10). Because of the weight function $\widehat{g}(s)$ in (5), the contribution from the horizontal lines of integration in the new contour is arbitrarily small. On the new vertical line of integration we use first use the bounds

$$
L\left(2 s, \chi_{E} \lambda_{E / K}^{2 \overrightarrow{\mathbf{m}}}\right)^{\beta(C, \chi, \mathbf{z})} \ll L^{A}
$$

for some $A>0$, which hold if $c_{6}$ is sufficiently small. As in [27] this follows from a Richert-type bound

$$
L\left(s, \chi_{E} \lambda_{E / K}^{\overrightarrow{\mathbf{m}}}\right) \ll W^{c_{7}(1-\sigma)^{3 / 2}} L^{2 / 3}
$$

for $\operatorname{Re} s>1-c_{8},|t| \leq W$ and $\|\overrightarrow{\mathbf{m}}\| \leq W$ (see [2]), and an application of the Borel-Caratheodory Theorem to $\log L\left(s, \chi_{E} \lambda_{E / K}^{\overrightarrow{\mathbf{m}}}\right)$.

For the factor $L\left(s, \lambda^{\overrightarrow{\mathbf{m}}}\right)$ in (10) we use the functional equation

$$
L\left(s, \lambda^{\overrightarrow{\mathbf{m}}}\right)=A^{1-2 s} G\left(s, \lambda^{\overrightarrow{\mathbf{m}}}\right) L\left(1-s, \bar{\lambda}^{\overrightarrow{\mathbf{m}}}\right) .
$$

Here $A=\left|d_{K}\right| \pi^{-n_{K}} 2^{-r_{2}}$ and $G$ is a quotient of gamma functions satisfying $G\left(s, \lambda^{\overrightarrow{\mathbf{m}}}\right) \ll W^{n\left(\frac{1}{2}-\sigma\right)}$ for the present range of $|t| \leq W$ and $\|\overrightarrow{\mathbf{m}}\| \leq W$. After switching $L\left(\frac{1}{2}-\eta+i t, \lambda^{\overrightarrow{\mathbf{m}}}\right)$ to $L\left(\frac{1}{2}+\eta-i t, \bar{\lambda}^{\overrightarrow{\mathbf{m}}}\right)$, we apply a fourth power moment result. (Such a result on the line $\operatorname{Re} s=1 / 2+\eta$, as opposed to $\operatorname{Re} s=1 / 2$, can be given by a method of Ramachandra's. See the proof of Lemma 10 of [3], for similar results.) Thus the contribution from the new vertical line of integration in (5) will be

$$
\begin{aligned}
& \ll a_{\overrightarrow{\mathbf{0}}} \ell x^{1 / 2-\eta} W^{n_{K} \eta} W^{n_{K}} L^{C} \ll x^{1 / 2}\left(\frac{\ell}{\Delta}\right)^{n_{K}}\left(\frac{W^{n_{K}}}{x}\right)^{\eta} L^{D} \\
& \ll x^{1 / 2}\left(\frac{\ell}{\Delta}\right)^{n_{K}}\left(\frac{1}{\Delta^{n_{K} x}}\right)^{\eta} L^{E} .
\end{aligned}
$$

When $s$ is near $1 / 2$ we can write

$$
F(s, \overrightarrow{\mathbf{0}}, \mathbf{z})=\left(\frac{1}{s-1 / 2}\right)^{\beta(\mathbf{z})-1} H_{0}(s, \mathbf{z}),
$$


with $H_{0}(s, \mathbf{z})$ analytic in some small disc around $s=1 / 2$, of radius $3 c_{8}$, say, and all $\mathbf{z}$ in Theorem 1 . Then the integral around $\mathcal{B}$ can be evaluated in a manner similar to (9) above. The leading term of the resulting sum is

$$
\begin{aligned}
a \overrightarrow{\mathbf{0}} & H_{0}(1 / 2, \mathbf{z}) \int \frac{g(y)}{y^{1 / 2} \log ^{2-\beta(\mathbf{z})} y} d y \\
& =\frac{(2 \ell)^{n_{K}} x^{1 / 2} H_{0}(1 / 2, \mathbf{z})}{\log ^{2-\beta(\mathbf{z})} x}\left(1+O(\ell)+O\left(\frac{\Delta}{\ell}\right)\right) .
\end{aligned}
$$

Only if $\Delta^{n_{K}}>x^{-\eta /(1+\eta)}$ can (12) dominate (11). Then we can recover an asymptotic result for the weighted sum (5) which includes explicitly the contributions from the poles of (10) at $s=1 / 2$. Unfortunately, if we strip the weights from (5), then we introduce an error of $O\left(x \ell^{n_{K}-1} \Delta\right.$ ) (assuming $\|\mathbf{z}\| \leq 1)$. To ensure that this error is smaller than (12), we have to take $\Delta$ so small that (11) dominates everything.

Instead, we accept that the poles of (10) at $s=1 / 2$ will contribute to the error in our final result. We choose $\Delta$ by equating (11) with $x \ell^{n_{K}-1} \Delta$, to get

$$
\Delta=\ell^{1 /(n+1+n \eta)} x^{-(1+2 \eta) / 2(n+1+n \eta)} .
$$

It is quickly checked that for such $\Delta$, the contribution from (11) dominates (12). We also require $\Delta \leq \ell$, which certainly holds for $\ell \geq x^{-1 / 2 n_{K}} x^{-\eta / 4 n_{K}}$, say. Thus we obtain

THEOREM 2 Let $f_{i}=\Omega_{i}-\omega_{i}$ for all $1 \leq i \leq t$. For $\|\mathbf{z}\| \leq 1$ and $x^{-1 / 2 n_{K}} \exp (-R(x))<\ell \leq 1 / 2$ we have

$$
\sum_{\mathfrak{a} \in \mathcal{S}(x, \psi, \ell)} \mathbf{z}^{\mathbf{f}(\mathfrak{a})}=x(2 \ell)^{n_{K}} H(1, \mathbf{z})+O\left(x^{1-\frac{1}{2(n+1)}} \ell^{n-1+\frac{1}{n+1}} \exp (-R(x))\right) .
$$

If $\ell=1 / 2$ and $\mathbf{z}=\mathbf{1}$, the error is weaker than we might expect, namely $O\left(x^{1-1 / n_{K}}\right)$, but, nonetheless, the main term dominates for $\ell>x^{-1 / 2 n_{K}} \exp$ $(-R(x))$, which is an improvement on Theorem 1.

2 Applications In the first two examples we set $\mathbf{z}=\mathbf{1}$ in Theorem 1 
2.1 Norms of ideals Let $F$ be any number field extension of $K$. For the integral ideals $\mathfrak{a}$ of $K$ define

$$
\theta_{1}(\mathfrak{a})= \begin{cases}1, & \text { if } \mathfrak{a} \text { is the relative norm of a fractional ideal of } F \\ 0, & \text { otherwise }\end{cases}
$$

and

$$
\theta_{2}(\mathfrak{a})= \begin{cases}1, & \text { if } \mathfrak{a} \text { is the relative norm of an integral ideal of } F \\ 0, & \text { otherwise. }\end{cases}
$$

It is easily checked that these functions are multiplicative (see, for example, Lemma 1.1 of [20]).

Recall that in our definition of $\mathcal{S}(x, \psi, \ell)$ we restricted to ideals that did not ramify in $L / K$. For such primes, it is easy to check that $\theta_{1}(\mathfrak{p})=1$ if, and only if, $\mathfrak{p} \mathcal{O}_{F}=\prod_{i=1}^{g} \mathcal{P}_{i}$ in $F$, where the $\mathcal{P}_{i}$ satisfy $N_{F / K} \mathcal{P}_{i}=\mathfrak{p}^{h_{i}}$ with $h_{i}$, for $i=1,2, \ldots, g$, collectively co-prime, while $\theta_{2}(\mathfrak{p})=1$ if, and only if, some $h_{i}=1$ (see for example Lemma 1.2 of [20]). These conditions on $h_{i}$ can be controlled by Proposition 2.8 of [11].

Let $L / K$ be the Galois closure of $F / K$ and let $H \leq G=\operatorname{Gal}(L / K)$ be the subgroup that fixes $F$ elementwise. Suppose $\sigma \in[(L / K) / \mathfrak{p}]$ has cycles of length $h_{1}, \ldots, h_{s}$ when acting upon the cosets of $H$ in $G$, then $\mathfrak{p}$ decomposes in $F$ as $\mathfrak{p}=\prod_{i=1}^{s} \mathcal{P}_{i}$ with $N_{L / K} \mathcal{P}_{i}=\mathfrak{p}^{h_{i}}$.

Of course, the cycle structure of $\sigma$ depends only on the conjugacy class containing it; hence $\theta_{1}$ and $\theta_{2}$ are Frobenius functions with respect to $L / K$. Thus we obtain asymptotic expansions of the form (2) for both the number of integral ideals in $K$, unramified in $F$, that are the relative norms of either fractional or integral ideals from $F$. In both cases, without further knowledge of particular examples of $F$ and $K$ we can say little other than the results hold for $\ell>x^{-3(1-\varepsilon) / 2\left(n_{L}+3 n_{K}\right)}$, where $n_{L}=\operatorname{deg} L / \mathbb{Q}$, unless the extension is abelian when we have $\ell>x^{-5(1-\varepsilon) / 12 n_{K}}$. The result for $\theta_{1}$ is then a localized form of the analogue in $L / K$ of Theorem IIA of [20]. The result for $\theta_{2}$ was promised in [4]. 
2.2 Modular Forms The examples above are special cases of the following situation considered by Serre in [24]. Let $g$ be a multiplicative function defined on the ideals of $K$ such that there exists a Galois extension $L$ of $K$ such that

$$
\{\mathfrak{p}: \mathfrak{p} \text { unramified in } L / K \text { and } g(\mathfrak{p})=0\}=\left\{\mathfrak{p}:\left[\frac{L / K}{\mathfrak{p}}\right] \in \bigcup_{r \in \mathcal{R}} C_{r}\right\},
$$

for some union of conjugacy classes in $\operatorname{Gal}(L / K)$. Define

$$
\widehat{g}(\mathfrak{p})= \begin{cases}1, & \text { if } g(\mathfrak{p}) \neq 0 \\ 0, & \text { if } g(\mathfrak{p})=0\end{cases}
$$

which is then a multiplicative Frobenius function. Thus we can apply Theorem 1 with $\mathbf{z}=\mathbf{1}$ and $\theta=\widehat{g}$ to obtain results on $\#\{\mathfrak{a} \in \mathcal{S}(x, \psi, \ell), g(\mathfrak{a}) \neq 0\}$. The main term will be of the form $c(2 \ell)^{n} x /(\log x)^{\beta}$ for some constant $c$, where $\beta=\sum_{r \in \mathcal{R}}\left|C_{r}\right| /|G|$ is thus a measure of the density of prime ideals $\mathfrak{p}$ for which $g(\mathfrak{p})=0$.

For a particular application, we examine Ramanujan's $\tau$-function defined formally by $\sum_{n \geq 1} \tau(n) q^{n}=q \prod_{m \geq 1}\left(1-q^{m}\right)^{24}$. If we write restrict to $q=$ $e^{2 \pi i z}$, with $\operatorname{Im} z>0$, then $f(z)=\sum_{n \geq 1} \tau(n) q^{n}$ is a modular form of weight 12. Let $m$ be a rational prime. As in [24], it is known that there exists a field extension $K_{m}$ of $\mathbb{Q}$ and an irreducible two-dimensional complex linear representation $\rho: \operatorname{Gal}\left(K_{m} / \mathbb{Q}\right) \rightarrow G L_{2}(\mathbb{Z} / m \mathbb{Z})$ such that, given a prime $p$ unramified in $K_{m}, \operatorname{Tr} \rho\left(\left[\left(K_{m} / \mathbb{Q}\right) / p\right]\right) \equiv \tau(p) \bmod m$. This shows that, outside the integers that ramify in $K_{m}$, the arithmetic function $\tau \bmod m$ is a multiplicative Frobenius function. Further, if $m>691$ the map $\rho$ is a bijection and so $\operatorname{deg} K_{m}=\left(m^{2}-1\right)\left(m^{2}-m\right)$. Thus we obtain

COROLLARY 1 For a prime $m>691$ and

$$
1>\frac{\log h}{\log x}>1-\frac{3}{2\left(\left(m^{2}-1\right)\left(m^{2}-m\right)+3\right)}
$$

it follows that

$$
\sharp\{x<n<x+h: \tau(n) \not \equiv 0(\bmod m)\}
$$


has an asymptotic expansion in the manner of (2) with main term

$$
\frac{c_{0} h}{\Gamma(1-\beta)(\log x)^{\beta}},
$$

for some constant $c_{0}$ independent of $m$ and where $\beta=m /\left(m^{2}-1\right)$.

PROOF We need only check the exponent of the logarithm which, because of our bijection, we can do by counting the number of elements of $G L_{2}(\mathbb{Z} / m \mathbb{Z})$ with trace 0 . There are $m^{2}(m-1)$ such elements so $\beta=m^{2}(m-1) /\left(m^{2}-\right.$ 1) $\left(m^{2}-m\right)=m /\left(m^{2}-1\right)$ as required.

2.3 Counting prime divisors Define $\mathbb{N}_{\infty}=\mathbb{N}_{0} \cup\{\infty\}$, where the symbol $\infty$ is simply a notational device so that, if $\mathbf{k} \in \mathbb{N}_{\infty}^{t}$, then we can write $\mathbf{f}(\mathfrak{a})=\mathbf{k}$ to mean $f_{i}(\mathfrak{a})=k_{i}$ if $k_{i} \neq \infty$, and there is no condition on $f_{i}(\mathfrak{a})$ if $k_{i}=\infty$. Then, for each $\mathbf{k} \in \mathbb{N}_{\infty}^{t}$ define

$$
v_{\mathbf{k}}(x, \psi, \ell)=\sum_{\substack{\mathfrak{a} \in \mathcal{S}(x, \psi, \ell) \\ \mathfrak{a} \text { integral } \\ \mathbf{f}(\mathfrak{a})=\mathbf{k}}} \theta(\mathfrak{a}),
$$

where $\theta$ satisfies the conditions in Theorem 1 . Let $\mathcal{H}=\mathcal{H}(\mathbf{k})=\left\{i: k_{i} \neq \infty\right\}$. If $\mathcal{H}=\emptyset$ then $v_{\mathbf{k}}(x, \psi, \ell)$ is simply the $t=1, z=1$ special case of Theorem 1 . So we assume that $\mathcal{H}$ is non-empty. For each $i \in \mathcal{H}$, we enforce the condition $f_{i}(\mathfrak{a})=k_{i}$ by the standard method of integrating the variable $z_{i}$ in (2) along a circle about the origin. For instance, start with the $J=0$ case of (2). This contains the term $\alpha(\mathbf{z})=\sum_{C}|C| \theta(C) z_{C} /|G|$, where $z_{C}=\prod_{i: f_{i}(\mathfrak{p}) \neq 0} z_{i}^{f_{i}(\mathfrak{p})}$ for any prime ideal $\mathfrak{p}$ with $\left[\frac{L / K}{\mathfrak{p}}\right]=C$. But, for any such $\mathfrak{p}$, we have $\Theta(\mathfrak{p})=\gamma_{j}$ for some $1 \leq j \leq t$. So $f_{i}(\mathfrak{p})=0$ for all $i \neq j$ and $f_{j}(\mathfrak{p})=1$ if $f_{j}=\omega_{j}$ or $\Omega_{j}$, zero otherwise. Hence $z_{C}=z_{j}$ if $f_{j}=\omega_{j}$ or $\Omega_{j}$, while $z_{C}=1$ if $f_{j}=\Omega_{j}-\omega_{j}$. Let $\left\{C_{i j}\right\}$ comprise all the conjugacy classes containing prime ideals $\mathfrak{p}$ with $\Theta(\mathfrak{p})=\gamma_{j}$, and set $\delta_{j}=\sum_{i}\left|C_{i j}\right| \theta\left(C_{i j}\right) /|G|$, a weighted density of such primes. Then

$$
\alpha(\mathbf{z})=\sum_{j: f_{j} \neq \Omega_{j}-\omega_{j}} \delta_{j} z_{j}+\sum_{j: f_{j}=\Omega_{j}-\omega_{j}} \delta_{j}
$$


If $i \notin \mathcal{H}$, then we are not enforcing a condition on $f_{i}(\mathfrak{a})$ and so we put $z_{i}=1$ in our instance of Theorem 1 . We will use the notation that, given any $t$-tuple $\mathbf{z}$, then $\mathbf{z}_{0}$ is the $t$-tuple given by $z_{0 i}=1 i \notin \mathcal{H}$ and $z_{0 i}=z_{i}$ if $i \in \mathcal{H}$. Our instance of Theorem 1 now contains the term $\alpha\left(\mathbf{z}_{0}\right)=\alpha+\sum_{j \in \mathcal{H}: f_{j} \neq \Omega_{j}-\omega_{j}} \delta_{j} z_{j}$, where

$$
\alpha=\sum_{\substack{j \notin \mathcal{H} \\ f_{j} \neq \Omega_{j}-\omega_{j}}} \delta_{j}+\sum_{j: f_{j}=\Omega_{j}-\omega_{j}} \delta_{j} .
$$

For simplicity define $\delta_{j}^{\prime}=\delta_{j}$ if $f_{j} \neq \Omega_{j}-\omega_{j}$, and zero otherwise. Now multiply both sides of the $J=0$ case of $(2)$ by $\prod_{i \in \mathcal{H}} z_{i}^{-\left(k_{i}+1\right)}$ and integrate over $\left|z_{i}\right|=r_{i}$ for each $i \in \mathcal{H}$, with $r_{i}<A_{i}$ to be chosen. Then the integral of the main term contains the factor

$$
\begin{aligned}
\int_{\left|z_{i}\right|=r_{i}} \ldots \int_{\log ^{1-\alpha\left(\mathbf{z}_{0}\right)} x} \frac{c_{0}\left(\mathbf{z}_{0}\right)}{\prod_{i \in \mathcal{H}}} \frac{d z_{i}}{z_{i}^{k_{i}+1}} \\
\quad=\frac{1}{\log ^{1-\alpha} x} \int_{\left|z_{i}\right|=\int_{i}} \ldots c_{0}\left(\mathbf{z}_{0}\right) \exp \left(\sum_{j \in \mathcal{H}} \delta_{j}^{\prime} X z_{j}\right) \prod_{i \in \mathcal{H}} \frac{d z_{i}}{z_{i}^{k_{i}+1}} \\
\quad=\frac{1}{\log ^{1-\alpha} x} \int_{\left|z_{i}\right|=\int_{i}} \ldots \int_{0} c_{0}\left(\mathbf{z}_{0}\right) \prod_{i \in \mathcal{H}} \frac{\exp \left(\delta_{j}^{\prime} X z_{j}\right)}{z_{i}^{k_{i}+1}} d z_{j}=\frac{P(X)}{\log ^{1-\alpha} x},
\end{aligned}
$$

where $X=\log \log x$. To describe this polynomial $P(y)$ further we require extra notation.

- Given any $\mathbf{v}=\left(v_{1}, \ldots, v_{t}\right) \in \mathbb{N}_{\infty}^{t}$, we write $\widetilde{\mathbf{v}}=\left(v_{i}\right)_{i \in \mathcal{H}} \in \mathbb{N}_{0}^{m}$ where $m=\# \mathcal{H}$. Also, we will write $\widetilde{v}$ and $\|\widetilde{\mathbf{v}}\|_{\sigma}$, interchangeably, for $\sum_{i \in \mathcal{H}} v_{i}$

- Let $\widetilde{\mathbf{a}}=\left(\delta_{i}^{\prime}\right)_{i \in \mathcal{H}}$, which is not derived from a $t$-tuple, but we use a tilde to show that it is indexed over $\mathcal{H}$.

- Let $\mathbf{b}=\mathbf{b}(\mathbf{k}) \in \mathbb{N}_{0}^{t}$ be defined by $b_{i}=0$ if $i \in \mathcal{H}$ and 1 if $i \notin \mathcal{H}$. Then $\alpha(\mathbf{b})=\alpha$. 
- Write $\widetilde{a}(\widetilde{\mathbf{z}})=\sum_{j \in \mathcal{H}} \delta_{j}^{\prime} z_{j}$.

- Finally, given $\widetilde{\mathbf{k}}=\left(k_{i}\right)_{i \in \mathcal{H}} \in \mathbb{N}_{0}^{m}$, we write $c_{\widetilde{\mathbf{k}}}=\widetilde{k} ! / \prod_{i \in \mathcal{H}} k_{i} !$, the multinomial coefficient.

Then

$$
P(y)=\sum_{\substack{\widetilde{\widetilde{n}} \\ 0 \leq n_{i} \leq k_{i}, i \in \mathcal{H}, \delta_{i}>0}} \frac{c_{\widetilde{\mathbf{n}}} c_{\widetilde{\mathbf{k}}-\widetilde{\mathbf{n}}} \partial^{\widetilde{\mathbf{n}}} c_{0}(\mathbf{b})}{\widetilde{n} !(\widetilde{k}-\widetilde{n}) !} \prod_{j \in \mathcal{H}}\left(\delta_{j}^{\prime} y\right)^{k_{j}-n_{j}},
$$

where $\partial^{\tilde{\mathbf{n}}} c_{0}(\mathbf{z})$ denotes the partial derivative of $c_{0}(\mathbf{z})$ at each $z_{i}, i \in \mathcal{H}$, of order $n_{i}$. We can see that $\operatorname{deg} P \leq \sum\left\{k_{i}: i \in \mathcal{H}, f_{i} \neq \Omega_{i}-\omega_{i}, \delta_{i} \neq 0\right\} \leq \widetilde{k}$. For simplicity we will assume that, if $0<k_{i}<\infty$, then $f_{i} \neq \Omega_{i}-\omega_{i}$ and $\delta_{i} \neq 0$. In this case, $\sum\left\{k_{i}: i \in \mathcal{H}, f_{i} \neq \Omega_{i}-\omega_{i}, \delta_{i} \neq 0\right\}=\widetilde{k}$. The first few terms of $P(y)$ are

$$
\begin{aligned}
P(y) & =\frac{c_{\widetilde{\mathbf{k}}} \widetilde{\mathbf{a}}^{\widetilde{\mathbf{k}}}}{\widetilde{k} !} c_{0}(\mathbf{b}) y^{\widetilde{k}}+\frac{c_{\widetilde{\mathbf{k}}} \widetilde{\mathbf{a}}^{\widetilde{\mathbf{k}}}}{\widetilde{k} !} \sum_{\substack{j \in \mathcal{H} \\
k_{j}>0}} \frac{k_{j}}{\delta_{j}} \partial_{x_{j}} c_{0}(\mathbf{b}) y^{\widetilde{k}-1}+\ldots \\
& =\frac{c_{\widetilde{\mathbf{k}}} \widetilde{\mathbf{a}^{\mathbf{k}}}}{\widetilde{k} !} \alpha U(\mathbf{b}) y^{\widetilde{k}}+\frac{c_{\widetilde{\mathbf{k}}} \widetilde{\mathbf{a}^{\mathbf{k}}}}{\widetilde{k} !}\left(\widetilde{k} U(\mathbf{b})+\left.\alpha \frac{d}{d t} U(\mathbf{v}(t))\right|_{t=0}\right) y^{\widetilde{k}-1}+\ldots
\end{aligned}
$$

where $\mathbf{v}(t)_{i}=1$ if $i \notin \mathcal{H}, t k_{i} / \delta_{i}^{\prime}$ if $i \in \mathcal{H}$ and $k_{i}>0$, and zero otherwise. Note that the second term dominates the first if $\widetilde{k} \geq \alpha y$. Since $\mathbf{k}$ satisfies the above assumption then $\widetilde{\mathbf{a}}^{\widetilde{\mathbf{k}}}=\prod_{i \in \mathcal{H}} \delta_{i}^{\prime k_{i}}$ is well-defined and non-zero (under the convention that $0^{0}=1$ ). This is because, if $\delta_{i}^{\prime}=0$ for some $i \in \mathcal{H}$, which can only happen if either $f_{i}=\Omega_{i}-\omega_{i}$ or $\delta_{i}=0$, then $k_{i}=0$ by our assumption. Hence $\operatorname{deg} P=\widetilde{k}$ if $\alpha \neq 0$ and $\operatorname{deg} P=\widetilde{k}-1$ if $\alpha=0$.

With the choice of $r_{i}=k_{i} /\left(\left|\delta_{i}\right| X\right)$ for each $i \in \mathcal{H}$, the error from the $J=0$ case of $(2)$ contributes

$$
\ll \frac{x \ell^{n_{K}}}{\log ^{2-\alpha} x} \prod_{i \in \mathcal{H}} \frac{1}{r_{i}^{k_{i}}} \int_{0}^{2 \pi} \exp \left(\delta_{i}^{\prime} X r_{i} \cos \theta\right) d \theta \ll \frac{x \ell^{n_{K}}}{\log ^{2-\alpha} x} \prod_{i \in \mathcal{H}} \frac{\left(\left|\delta_{i}^{\prime}\right| X\right)^{k_{i}}}{k_{i} !},
$$

having used the $n=0$ case of

$$
\int_{0}^{2 \pi}\left|e^{i \theta}-1\right|^{n} \exp (y \cos \theta) d \theta \ll_{n} e^{y} y^{-\frac{n+1}{2}}
$$


(See [26] p. 204 for a proof.) Hence we have

THEOREM 3 Let $\mathbf{k} \in \mathbb{N}_{\infty}^{t}$ be such that if $0<k_{i}<\infty$ then $f_{i} \neq \Omega_{i}-\omega_{i}$ and $\delta_{i} \neq 0$. Then subject to the conditions on $\ell$ in Theorem 1 ,

$$
v_{\mathbf{k}}(x, \psi, \ell)=\frac{x(2 \ell)^{n_{K}}}{\log ^{1-\alpha} x} P(\log \log x)+O\left(\frac{x \ell^{n_{K}}}{\log ^{2-\alpha} x} \prod_{i \in \mathcal{H}} \frac{\left(\left|\delta_{i}\right| \log \log x\right)^{k_{i}}}{k_{i} !}\right)
$$

valid for $k_{i} \leq A_{i}\left|\delta_{i}\right| \log \log x$ for each $i \in \mathcal{H}$.

If we assume $\theta \geq 0$, then an alternative choice of the $r_{i}$ gives

THEOREM 4 Let $\mathbf{k} \in \mathbb{N}_{\infty}^{t}$ be such that if $0<k_{i}<\infty$ then $f_{i} \neq \Omega_{i}-\omega_{i}$ and $\delta_{i} \neq 0$ and assume $\theta \geq 0$. Then, subject to the conditions on $\ell$ in Theorem 1 ,

$$
\begin{aligned}
v_{\mathbf{k}}(x, \psi, \ell)= & \frac{x(2 \ell)^{n_{K}}}{\log ^{1-\alpha} x}\left\{\frac{c_{\widetilde{\mathbf{k}}} \widetilde{\mathbf{a}}^{\widetilde{\mathbf{k}}}}{\widetilde{k} !} \alpha(\log \log x)^{\widetilde{k}}+\frac{c_{\widetilde{\mathbf{k}}} \widetilde{\mathbf{a}}^{\widetilde{\mathbf{k}}}}{(\widetilde{k}-1) !}(\log \log x)^{\widetilde{k}-1}\right\} \\
& \times\left(U(\mathbf{r})+O\left(\frac{\widetilde{k}}{(\log \log x)^{2}}\right)\right)
\end{aligned}
$$

where $\mathbf{r}$ is defined by

$$
r_{i}=\left\{\begin{array}{cl}
1, & \text { if } k_{i}=\infty, \\
B k_{i} /\left(\delta_{i} X\right), & \text { if } k_{i}<\infty,
\end{array}\right.
$$

and $B=(\alpha \log \log x+\widetilde{k}-1) /(\alpha \log \log x+\widetilde{k})$.

PROOF In the integrand of (13) we write $c_{0}\left(\mathbf{z}_{0}\right)=\alpha\left(\mathbf{z}_{0}\right) U\left(\mathbf{z}_{0}\right)$ and expand

$$
\begin{aligned}
U\left(\mathbf{z}_{0}\right)= & U(\mathbf{r})+\sum_{i \in \mathcal{H}}\left(z_{i}-r_{i}\right) \partial_{x_{i}} U(\mathbf{r}) \\
& +\sum_{i, j \in \mathcal{H}}\left(z_{i}-r_{i}\right)\left(z_{j}-r_{j}\right) \int_{0}^{1}(1-s) \partial_{x_{i}, x_{j}}^{2} U\left(\mathbf{r}+s\left(\mathbf{z}_{0}-\mathbf{r}\right)\right) d s
\end{aligned}
$$

We introduce the notation

$$
I_{j}(k+1)=\frac{1}{2 \pi} \int_{\left|z_{i}\right|=r_{i}} \frac{(\log x)^{\delta_{j} z_{j}}}{z_{j}^{k_{j}+1}} d z_{j}=\frac{\left(\delta_{j} X\right)^{k_{j}}}{k_{j} !} .
$$


The contribution of the first term from (16) to the integrals in (13) is

$$
\begin{aligned}
& U(\mathbf{r})(\log x)^{\alpha} \int_{\left|z_{i}\right|=r_{i}, i \in \mathcal{H}} \ldots \int_{i \in \mathcal{H}}\left(\alpha+\sum_{i} \delta_{i} z_{i}\right) \prod_{j \in \mathcal{H}} \log ^{\delta_{j} z_{j}} x \frac{d z_{j}}{z_{j}+1} \\
& =U(\mathbf{r})(\log x)^{\alpha}\left(\alpha \prod_{j \in \mathcal{H}} I_{j}\left(k_{j}+1\right)+\sum_{i \in \mathcal{H}} \delta_{i} \prod_{\substack{j \in \mathcal{H} \\
j \neq i}} I_{j}\left(k_{j}+1\right) I_{i}\left(k_{i}\right)\right) \\
& =U(\mathbf{r})(\log x)^{\alpha} \prod_{j \in \mathcal{H}} I_{j}\left(k_{j}+1\right)\left(\alpha+\sum_{i \in \mathcal{H}} \frac{k_{i}}{X}\right)
\end{aligned}
$$

since $\delta_{i} X I_{i}(k)=k I_{i}(k+1)$. Thus we get the main term in (15). Below we will refer to the Main term as M.T. The introduction of a factor $z_{i}-r_{i}$, arising from the first sum on the right hand side of (16) will give a contribution to the integral of $(\log x)^{\alpha} \partial_{x_{i}} U(\mathbf{r})$ times

$$
\begin{aligned}
& \alpha\left\{\prod_{\substack{j \in \mathcal{H} \\
j \neq i}} I_{j}\left(k_{j}+1\right) I_{i}\left(k_{i}\right)-r_{i} \prod_{j \in \mathcal{H}} I_{j}\left(k_{j}+1\right)\right\} \\
& \quad+\sum_{n \neq i} \delta_{n}\left\{\prod_{\substack{j \in \mathcal{H} \\
j \neq i, n}} I_{j}\left(k_{j}+1\right) I_{i}\left(k_{i}\right) I_{n}\left(k_{n}\right)-r_{i} \prod_{\substack{j \in \mathcal{H} \\
j \neq n}} I_{j}\left(k_{j}+1\right) I_{n}\left(k_{n}\right)\right\} \\
& +\delta_{i}\left\{\prod_{\substack{j \in \mathcal{H} \\
j \neq i}} I_{j}\left(k_{j}+1\right) I_{i}\left(k_{i}-1\right)-r_{i} \prod_{\substack{j \in \mathcal{H} \\
j \neq i}} I_{j}\left(k_{j}+1\right) I_{i}\left(k_{i}\right)\right\},
\end{aligned}
$$

with the convention that $I_{i}(k)=0$ if $k \leq 0$. With the choice of $r_{i}$ given in the theorem this contribution is seen to be zero.

For the final term in $(16)$ we use $\partial_{x_{i}, x_{j}}^{2} U\left(\mathbf{r}+s\left(\mathbf{z}_{0}-\mathbf{r}\right)\right) \ll 1$. If we first forget the factors $\left|z_{i}-r_{i}\right|\left|z_{j}-r_{j}\right|$ in the double sum in (16), then each term contributes

$$
\begin{aligned}
& \ll \frac{x(2 \ell)^{n_{K}}}{\log ^{1-\alpha} x}\left(\alpha \prod_{j \in \mathcal{H}} J_{j}\left(k_{j}+1\right)+\sum \delta_{i} \prod_{\substack{j \in \mathcal{H} \\
j \neq i}} J_{j}\left(k_{j}+1\right) J_{i}\left(k_{i}\right)\right) \\
& \ll M . T . \times \frac{\exp ((B-1) \widetilde{k})}{B^{\widetilde{k}+t / 2}} \ll_{t} M \cdot T .
\end{aligned}
$$


Here we have written

$$
J_{j}(k+1)=\frac{1}{r_{j}^{k}} \int_{0}^{2 \pi} \exp \left(\delta_{j} X r_{i} \cos \theta\right) d \theta \ll \frac{\exp \left(\delta_{j} r_{j} X\right)}{r_{j}^{k} \sqrt{\delta_{j} r_{j} X}},
$$

by (14). With the factor $\left|z_{i}-r_{i}\right|\left|z_{j}-r_{j}\right|$ we immediately get a saving of $r_{i} r_{j}$ over the Main Term, along with the fact that either two of the integrals (in both terms of (17)) contain $\left|e^{i \theta}-1\right|$ or one contains $\left|e^{i \theta}-1\right|^{2}$. From (14) with either $n=1$ or $n=2$ we get an additional saving of $\ll 1 / \sqrt{\delta_{i} r_{i} X} \sqrt{\delta_{j} r_{j} X}$. Hence, for every term in the double sum in (16), we get a saving over the main Term of

$$
\frac{r_{i} r_{j}}{\sqrt{\delta_{i} r_{i} X} \sqrt{\delta_{j} r_{j} X}} \ll \frac{\sqrt{r_{i} r_{j}}}{X} \ll \frac{B \sqrt{k_{i} k_{j}}}{X} \ll \frac{\widetilde{k}}{X} .
$$

Finally, the choice of $r$ in the present result does not essentially change the estimate, given in the proof of Theorem 3, of the contribution from the error in Theorem 1.

In Theorem 6 below we will prove a result for $v_{\mathbf{k}}(x, \psi, \ell)$ valid for a longer range of $\widetilde{k}$ than Theorem 3 . First we show that Theorem 3 is sufficient to prove an Erdos-Kac type theorem.

THEOREM 5 Let $\mathcal{H}$ be a subset of $\{1, \ldots, t\}$ such that, if $i \in \mathcal{H}$, then $\delta_{i}>0$. For $i \in \mathcal{H}$, let $f_{i}=\omega_{i}$ or $\Omega_{i}$. Then, for any $\lambda_{i} \in \mathbb{R}, i \in \mathcal{H}$ and for $\ell$ satisfying the conditions of Theorem 1 ,

$$
\begin{aligned}
\frac{1}{\# \mathcal{S}(x, \psi, \ell)} \# & \left\{\mathfrak{a} \in \mathcal{S}(x, \psi, \ell): f_{i}(\mathfrak{a}) \leq \delta_{i} \log \log x+\lambda_{i} \sqrt{\delta_{i} \log \log x}\right\} \\
& \rightarrow \prod_{i \in \mathcal{H}} \Phi\left(\lambda_{i}\right)
\end{aligned}
$$

as $x \rightarrow \infty$ where

$$
\Phi(\lambda)=\frac{1}{\sqrt{2 \pi}} \int_{-\infty}^{\lambda} e^{-t^{2} / 2} d t
$$

PROOF We apply Theorem 3 with $\theta \equiv 1$, noting that $\delta_{j}^{\prime}=\delta_{j}$ for all $j \in \mathcal{H}$ and $1-\alpha=\sum_{j \in \mathcal{H}} \delta_{j}$ because of the assumption on $\mathcal{H}$. Hence, with $U_{i}(\lambda)=$ 
$\delta_{i} X+\lambda \sqrt{\delta_{i} X}$, we get

$$
\begin{aligned}
\frac{1}{x(2 \ell)^{n_{K}}} \sum_{k_{i} \leq U_{i}\left(\lambda_{i}\right)} v_{\mathbf{k}}(x, \psi, \ell)= & \sum_{\substack{\widetilde{\mathbf{n}}, \widetilde{\mathbf{m}} \\
0 \leq n_{i}+m_{i} \leq U_{i}\left(\lambda_{i}\right)}} \frac{c_{\widetilde{\mathbf{n}}} \partial^{\widetilde{\mathbf{n}}} c_{0}(\mathbf{b})}{\widetilde{n} !} \prod_{j \in \mathcal{H}} e^{-\delta_{j} X} \frac{\left(\delta_{j} X\right)^{m_{j}}}{m_{j} !} \\
& +O\left(\frac{1}{\log x} \prod_{j \in \mathcal{H}} E\left(\delta_{j} X, \lambda_{j}\right)\right)
\end{aligned}
$$

where the conditions on the summations are presumed to hold for all $i \in \mathcal{H}$, and

$$
E(y, \lambda):=\sum_{m \leq y+\lambda \sqrt{y}} e^{-y} \frac{y^{m}}{m !} .
$$

We know that $\sum_{\widetilde{\mathbf{n}}} c_{\widetilde{\mathbf{n}}} \partial^{\widetilde{\mathbf{n}}} c_{0}(\mathbf{b}) / \widetilde{n}$ ! converges, absolutely, to $c_{0}(\mathbf{1})$. In fact, $\partial^{\widetilde{\mathbf{n}}} c_{0}(\mathbf{b}) \ll 1$ for all $\widetilde{\mathbf{n}}$. Also

$$
E(y, \lambda)=\Phi(\lambda)+O\left(\frac{1}{\sqrt{y}}\right)
$$

as $y \rightarrow \infty$. So, in particular, the limit as $x \rightarrow \infty$ of the last term in (19) is zero. To calculate the limit of the first sum on the right hand side of (19) we replace the sum over $\widetilde{\mathbf{n}}, \widetilde{\mathbf{m}}: 0 \leq n_{i}+m_{i} \leq U_{i}\left(\lambda_{i}\right), i \in \mathcal{H}$ by one over $\widetilde{\mathbf{n}}, \widetilde{\mathbf{m}}: 0 \leq n_{i}, m_{i} \leq U_{i}\left(\lambda_{i}\right), i \in \mathcal{H}$. The error introduced, that is, the sum over $\widetilde{\mathbf{n}}, \widetilde{\mathbf{m}}: 0 \leq n_{i}+m_{i}>U_{i}\left(\lambda_{i}\right), i \in \mathcal{H}$, is split into two. Let $\varepsilon>0$ be given and set $Y=\varepsilon \sqrt{X}$. Take any $i \in \mathcal{H}$ and relabel as 1 . The first sum will be over $\widetilde{\mathbf{n}}$ for which $n_{1}<Y$ and the other over $\widetilde{\mathbf{n}}$ for which $n_{1} \geq Y$. In first sum $m_{1}>U_{1}\left(\lambda_{1}\right)-n_{1}>U_{1}\left(\lambda_{1}-\frac{\varepsilon}{\sqrt{\delta_{1}}}\right)$. Thus we can bound this sum by

$$
\begin{gathered}
\sum_{\substack{\tilde{\mathbf{n}} \\
0 \leq n_{i} \leq U_{i}\left(\lambda_{i}\right)}} \frac{c_{\widetilde{\mathbf{n}}}\left|\partial^{\widetilde{\mathbf{n}}} c_{0}(\mathbf{b})\right|}{\widetilde{n} !} \prod_{\substack{j \in \mathcal{H} \\
j \neq 1}} E\left(\delta_{j} X, \lambda_{j}\right) \sum_{\substack{m_{1}<U_{1}\left(\lambda_{1}\right) \\
m_{1}>U_{1}\left(\lambda_{1}-\frac{\varepsilon}{\sqrt{\delta_{1}}}\right)}} e^{-\delta_{1} X} \frac{\left(\delta_{1} X\right)^{m_{1}}}{m_{1} !} \\
\ll\left(\Phi\left(\lambda_{1}\right)-\Phi\left(\lambda_{1}-\frac{\varepsilon}{\sqrt{\delta_{1}}}\right)\right)+\frac{1}{\sqrt{X}} \ll \varepsilon
\end{gathered}
$$


for $x$ sufficiently large. The second sum is bounded by

$$
\begin{aligned}
& \ll\left(\sum_{\varepsilon \sqrt{X} \leq n_{1} \leq U_{1}\left(\lambda_{1}\right)} \frac{1}{n !}\right) \prod_{\substack{i \in \mathcal{H} \\
i \neq 1}}\left(\sum_{n_{i} \leq U_{i}\left(\lambda_{i}\right)} \frac{1}{n_{i} !}\right) \prod_{j \in \mathcal{H}} E\left(\delta_{j} X, \lambda_{j}\right) \\
& \ll \varepsilon
\end{aligned}
$$

for sufficiently large $x$. Hence the limit of the first sum on the right hand side of (19) equals

$$
\lim _{x \rightarrow \infty} \sum_{\substack{\widetilde{\mathbf{n}} \\ 0 \leq n_{i} \leq U_{i}\left(\lambda_{i}\right)}} \frac{c_{\widetilde{\mathbf{n}}} \partial^{\tilde{\mathbf{n}}} c_{0}(\mathbf{b})}{\widetilde{n} !} \prod_{j \in \mathcal{H}} E\left(\delta_{j} X, \lambda_{j}\right)=c_{0}(\mathbf{1}) \prod_{j \in \mathcal{H}} \Phi\left(\lambda_{j}\right) .
$$

Finally, from (2) we see that

$$
\lim _{x \rightarrow \infty} \frac{\# \mathcal{S}(x, \psi, l)}{x(2 \ell)^{n_{K}}}=c_{0}(\mathbf{1}) .
$$

Hence the result follows.

3 Further results for $v_{\mathbf{k}}(x, \psi, \ell)$ The following result is a generalization of Theorem 5 of [5], and the proof consists of indicating changes that need to be made in that earlier proof.

THEOREM 6 Assume that $|\theta| \leq 1$ and that $|\alpha(\mathbf{z})|<1$ for all $\mathbf{z}$ for which $\left|z_{j}\right|<1$ when $j \in \mathcal{H}$, and $\left|z_{j}\right| \leq 1$ when $j \notin \mathcal{H}$. Then, subject to the conditions on $\ell$ in Theorem 1 ,

$$
\begin{aligned}
v_{\mathbf{k}}(x, \psi, \ell)= & \frac{x(2 \ell)^{n_{K}}}{(\log x)^{1-\alpha}} \sum_{0 \leq j \leq J} \frac{P_{j, \mathbf{k}}(\log \log x)}{(\log x)^{j}} \\
& +x \ell^{n_{K}} E_{\mathbf{k}, J}(x)+O\left(x \ell^{n_{K}} \exp (-R(x))\right)
\end{aligned}
$$

for some polynomials $P_{j, \mathbf{k}}$ satisfying

$$
P_{j, \mathbf{k}}(\log \log x) \ll_{\alpha} \frac{\Gamma(j+2)}{\left(2 c_{0}\right)^{j}} \log x
$$

uniformly in $\mathbf{k}$, and where

$$
\begin{aligned}
E_{\mathbf{k}, J}(x) \ll \frac{1}{(\log x)^{1-\operatorname{Re} \alpha}}\left(\frac{(\log \log x)^{\widetilde{k}}}{\left(2 c_{0} \log x\right)^{J+1}}\left(\frac{\widetilde{k} !}{J}+J !\right)\right. \\
\left.+\frac{J+1}{\left(2 c_{0}\right)^{J}} x^{-c_{0}}\left(\log 1 / c_{0}\right)^{\widetilde{k}}+\ell \log \log ^{2} x\right),
\end{aligned}
$$


subject to $J+2 \leq c_{0} \log x$.

If $J=J(x)=\kappa_{2}(\log x)^{1 / 3}(\log \log x)^{-4 / 3}, k \leq C J(x)$ and $\ell \leq \exp (-R(x))$ then $x \ell^{n_{K}} E_{J} \ll x \ell^{n_{K}} \exp (-R(x))$.

PROOF In the proof of Theorem 1 a truncated asymptotic expansion was given for

$$
\frac{1}{2 \pi i} \int_{\mathcal{C}_{0}} y^{s-1} F(s, \overrightarrow{\mathbf{0}}, \mathbf{z}) d s .
$$

An alternative method to that in the proof of Theorem 1 is to replace $\mathcal{C}_{0}$ by the truncated Hankel contour $\mathcal{C}_{\delta}$ running from $1-c_{0}$ to $1-\delta$ with argument $-\pi$, then around 1 on a circular arc radius $\delta$ and finally from $1-\delta$ to $1-c_{0}$ with argument $\pi$. By the assumptions in the Theorem, $|\alpha(\mathbf{z})|<1$, and, in particular, $\operatorname{Re} \alpha(\mathbf{z})<1$, so that the contribution from the circular arc tends to zero as $\delta \rightarrow 0$. The integration over $y$ in (7) then gives, for the $\ell$ of Theorem 1, a generalization of Theorem 3 of [5], namely

$$
\begin{gathered}
\sum_{\substack{\mathfrak{a} \in \mathcal{S}(x, \psi, \ell) \\
\mathfrak{a} \text { integral }}} \theta(\mathfrak{a}) \mathbf{z}^{\mathbf{f}(\mathfrak{a})}=x(2 \ell)^{n_{K}} \int_{0}^{c_{0}} x^{-r} k(\ell, r) F^{*}(1-r, \mathbf{z}) r^{-\alpha(\mathbf{z})} d r \\
+O\left(x \ell^{n_{K}} \exp (-R(x))\right)
\end{gathered}
$$

where $k(\ell, r)=(2 \ell)^{-1}\left\{(1+\ell)^{1-r}-(1-\ell)^{1-r}\right\}$ and $F^{*}(s, \mathbf{z})=(\pi s)^{-1} H(s, \mathbf{z})$ $\sin (\pi \alpha(\mathbf{z}))$.

Put $z_{i}=1$ in (21) for each $i \notin \mathcal{H}$, multiply both sides by $\prod_{i \in \mathcal{H}} z_{i}^{-\left(k_{i}+1\right)}$, perform $m$ integrations over $\left|z_{i}\right|=1-\varepsilon_{i}$ and let each $\varepsilon_{i} \rightarrow 0$ to obtain

$$
\nu_{\mathbf{k}}(x, \psi, \ell)=x(2 \ell)^{n_{K}} \int_{0}^{c_{0}} x^{-r} k(\ell, r) W_{\widetilde{\mathbf{k}}}(r) r^{-\alpha} d r+O\left(x \ell^{n_{K}} \exp (-R(x))\right) .
$$

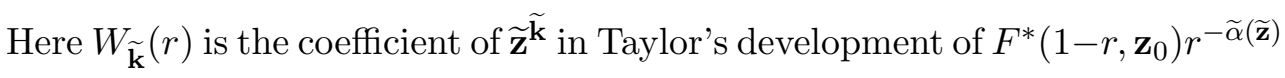
about $\widetilde{\mathbf{z}}=\widetilde{\mathbf{0}}$. Since $F^{*}(1-s, \mathbf{z})$ is analytic for $|s|<3 c_{0}$ and $\max \left|z_{i}\right| \leq 1$, we can write

$$
F^{*}\left(1-s, \mathbf{z}_{0}\right)=\sum_{j=0}^{\infty} \sum_{\widetilde{\mathbf{u}} \in \mathbb{N}_{0}^{m}} \beta_{j, \widetilde{\mathbf{u}}} \widetilde{\mathbf{z}}^{\tilde{u}^{j}}
$$


Just as we saw for the $h_{j}(\mathbf{z})$ in the proof of Theorem 1 , we have $\beta_{j, \widetilde{\mathbf{u}}} \ll$ $\left(2 c_{0}\right)^{-j}$ for all $j \geq 1$, uniformly in $\widetilde{\mathbf{u}}$. We also have

$$
\begin{aligned}
r^{-\widetilde{\alpha}(\widetilde{\mathbf{z}})} & =\sum_{p=0}^{\infty} \frac{\widetilde{a}(\widetilde{\mathbf{z}})^{p}}{p !}(-\log r)^{p} \\
& =\sum_{p=0}^{\infty} \frac{1}{p !}\left(\sum_{\|\widetilde{\mathbf{p}}\|_{\sigma}=p} c_{\widetilde{\mathbf{p}}} \widetilde{\mathbf{a}}^{\widetilde{\mathbf{p}}} \widetilde{\mathbf{z}}^{\widetilde{\mathbf{p}}}\right)(-\log r)^{p}
\end{aligned}
$$

by the multinomial theorem. Thus

$$
W_{\widetilde{\mathbf{k}}}(r)=\sum_{j=0}^{\infty} r^{j} \sum_{0 \leq p \leq \widetilde{k}} \frac{1}{p !}\left(\sum_{\substack{\tilde{\mathbf{u}}+\widetilde{\mathbf{p}}=\widetilde{\mathbf{k}} \\\|\widetilde{\mathbf{p}}\|_{\sigma}=p}} c_{\widetilde{\mathbf{p}}} \widetilde{\mathbf{a}}^{\widetilde{\mathbf{p}}} \beta_{j, \widetilde{\mathbf{u}}}\right)(-\log r)^{p} .
$$

Let $\beta_{j, \widetilde{\mathbf{k}}, p}$ denote the inner sum. With the assumptions on $\theta$, we have that $\left|a_{i}\right| \leq d_{i}$ for each $i$, where $d_{i}$ is the density of $\mathfrak{p}: \Theta(\mathfrak{p})=\gamma_{i}$. Write $\mathbf{d}=$ $\left(d_{i}\right)_{1 \leq i \leq t}$, then

$$
\begin{aligned}
\beta_{j, \widetilde{\mathbf{k}}, p} & \ll \frac{1}{\left(2 c_{0}\right)^{j}} \sum_{\substack{\widetilde{\mathbf{u}}+\widetilde{\mathbf{p}}=\mathbf{k} \\
\|\widetilde{\mathbf{p}}\|_{\sigma}=p}} c_{\widetilde{\mathbf{p}}} \widetilde{\mathbf{d}}^{\widetilde{\mathbf{p}}}=\frac{1}{\left(2 c_{0}\right)^{j}} \sum_{\|\widetilde{\mathbf{p}}\|_{\sigma}=p} c_{\widetilde{\mathbf{p}}} \widetilde{\mathbf{d}}^{\widetilde{\mathbf{p}}} \\
& =\frac{1}{\left(2 c_{0}\right)^{j}}\left(\sum_{i \in \mathcal{H}} d_{i}\right)^{p} \leq \frac{1}{\left(2 c_{0}\right)^{j}} .
\end{aligned}
$$

So, on writing

$$
W_{\widetilde{\mathbf{k}}}(r)=\sum_{j=0}^{\infty} r^{j} \sum_{0 \leq p \leq \widetilde{k}} \frac{\beta_{j, \widetilde{\mathbf{k}}, p}}{p !}(-\log r)^{p},
$$

we see that $W_{\widetilde{\mathbf{k}}}(r)$ is of exactly the same form as in [29], p.11, with the same information concerning the coefficients $\beta_{j, \widetilde{\mathbf{k}}, p}$.

We truncate the series over $j$ in (23) at some $J \geq 1$. The contribution of the resulting error to the integral (22) can be read from equation (13) in [5]. The truncated sum is inserted into (22) and the summation and integration interchanged. The integral is now extended from $c_{0}$ to $\infty$. The error in doing this can be read from equation (16) in [5]. (The inclusion of $r^{-\alpha}$ in (22) only effect the errors by reducing $J$ by at most 1 in equation (13) of [5].) Thus we get the first two terms in $E_{\mathbf{k}, J}$. 
For the integrals over $r$, from 0 to $\infty$, of each term $0 \leq j \leq J$ in the sum of (23) we first replace $k(\ell, r)$ by 1 . We then get a term

$$
M_{j}(x):=\sum_{0 \leq p \leq \widetilde{k}} \sum_{m=0}^{p} a_{p, m, j} \frac{(\log \log x)^{m}}{(\log x)^{j+1-\alpha}}
$$

where

$$
a_{p, m, j}=\frac{\beta_{j, \widetilde{\mathbf{k}}, p}(-1)^{p-m} \Gamma^{(p-m)}(j-\alpha+1)}{m !(p-m) !} .
$$

We incorporate the factor $k(\ell, r)$ into the integration by calculating

$$
\frac{1}{2 \ell}\left\{(1+\ell) M_{j}(x(1+\ell))-(1-\ell) M_{j}(x(1-\ell))\right\} .
$$

For each term in $M_{j}(x)$ we have, by a mean value result, that

$$
\begin{array}{r}
\frac{1}{2 \ell}\left\{\frac{(1+\ell)(\log \log (x(1+\ell)))^{m}}{(\log (x(1+\ell)))^{j+1-\alpha}}-\frac{(1-\ell)(\log \log (x(1-\ell)))^{m}}{(\log (x(1-\ell)))^{j+1-\alpha}}\right\} \\
=\frac{X^{m}}{\log ^{j+1-\alpha} x}+\frac{m X^{m-1}-(j-\alpha+1) X^{m}}{\log ^{j+2-\alpha} x} \\
+O\left(\left(j^{2}+m^{2}\right) \ell \frac{X^{m}}{\log ^{j+2-\operatorname{Re} \alpha} x}\right)
\end{array}
$$

where $X=\log \log x$. The contribution of this error to (24) is

$$
\ll \frac{\ell}{\left(2 c_{0}\right)^{j} \log ^{j+2-\operatorname{Re} \alpha} x} \sum_{m=0}^{\widetilde{k}} \frac{\left(j^{2}+m^{2}\right) X^{m}}{m !} \sum_{n=0}^{\widetilde{k}-m} \frac{\left|\Gamma^{(n)}(j-\alpha+1)\right|}{n !}
$$

We estimate $\left|\Gamma^{(n)}(j-\alpha+1)\right|$ by using Cauchy's integral on a circle about $j-\alpha+1$ of radius $(1-|\alpha|) / 2$. In such an integral the integrand contains

$$
\begin{aligned}
\Gamma(j-\alpha+1+w) & =(j-\alpha+w) \ldots(1-\alpha+w) \Gamma(1-\alpha+w) \\
& \ll \alpha(j+1) j \ldots 2=\Gamma(j+2)
\end{aligned}
$$

for all $|w|=(1-|\alpha|) / 2$, using $|\alpha|<1$. Thus $\left|\Gamma^{(n)}(j-\alpha+1)\right| \ll_{\alpha}((1-$ $|\alpha|) / 2)^{n} \Gamma(j+2)$ and, in particular, the sum over $n$ in (26) satisfies

$$
\sum_{n=0}^{\widetilde{k}-m} \frac{\left|\Gamma^{(n)}(j-\alpha+1)\right|}{n !} \ll_{\alpha} \Gamma(j+2) .
$$


Hence (26) is

$$
\begin{aligned}
& \ll \alpha \frac{\ell \Gamma(j+2)}{\left(2 c_{0}\right)^{j} \log ^{j+2-\operatorname{Re} \alpha} x} \sum_{m=0}^{\widetilde{k}} \frac{\left(j^{2}+m^{2}\right) X^{m}}{m !} \\
& \ll \alpha \frac{\ell \Gamma(j+2)}{\left(2 c_{0}\right)^{j} \log ^{j+2-\operatorname{Re} \alpha} x}\left(j^{2} \exp (X)+\left(X+X^{2} \exp (X)\right)\right) \\
& \ll \alpha \frac{\ell \Gamma(j+2)\left(j^{2}+X^{2}\right)}{\left(2 c_{0}\right)^{j} \log ^{j+1-\operatorname{Re} \alpha} x} .
\end{aligned}
$$

Summing over $j$ and assuming that $J+2 \leq c_{0} \log x$ gives the third term in $E_{\mathbf{k}, J}$, namely $\ell(\log \log x)^{2}$.

The main terms in (25) lead to a contribution in (24) of

$$
\frac{1}{\log x^{1-\alpha}} \sum_{0 \leq p \leq \widetilde{k}} \sum_{m=0}^{p} a_{p, m, j}\left(\frac{X^{m}}{\log ^{j} x}+m \frac{X^{m-1}}{\log ^{j+1} x}-(j-\alpha+1) \frac{X^{m}}{\log ^{j+1} x}\right),
$$

say. When we sum over $j$ we find that the polynomial coefficient of $(\log x)^{-j-1-\alpha}$, that is $P_{j, \mathbf{k}}(X)$, is given by

$$
\sum_{0 \leq p \leq \widetilde{k}} \sum_{m=0}^{p} b_{p, m, j} X^{m}=\sum_{m=0}^{\widetilde{k}}\left(\sum_{m \leq p \leq \widetilde{k}} b_{p, m, j}\right) X^{m}
$$

where

$$
\begin{array}{ll}
b_{p, m, 0}=a_{p, m, 0} & \text { for all } p \geq 0,0 \leq m \leq p, \\
b_{p, m, j}=a_{p, m, j}+(m+1) a_{p, m+1, j-1} & \\
-(j-\alpha) a_{p, m, j-1} & \text { for all } j \geq 1, p \geq 0,0 \leq m \leq p-1, \\
b_{p p j}=a_{p, p, j}-(j-\alpha) a_{p, p, j-1} & \text { for all } j \geq 1, p \geq 0 .
\end{array}
$$

Using (27) we find that

$$
\begin{aligned}
P_{j, \mathbf{k}}(X) & \ll \frac{1}{\left(2 c_{0}\right)^{j}} \sum_{m=0}^{\widetilde{k}} \frac{X^{m}}{m !}\left(\sum_{n=0}^{\widetilde{k}-m} \frac{\left|\Gamma^{(n)}(j-\alpha+1)\right|}{n !}+\sum_{n=0}^{\widetilde{k}-m-1} \frac{\left|\Gamma^{(n)}(j-\alpha)\right|}{n !}\right. \\
& \left.+(j+1) \sum_{n=0}^{\widetilde{k}-m} \frac{\left|\Gamma^{(n)}(j-\alpha)\right|}{n !}\right) \\
& \ll \alpha \frac{\Gamma(j+2)}{\left(2 c_{0}\right)^{j}} \exp (X),
\end{aligned}
$$


uniformly in $\mathbf{k}$.

NOTE (iii) The assumption on $\alpha(\mathbf{z})$ in Theorem 6 can only fail if $\theta \equiv 1$ and $\sum_{j \in \mathcal{H}, f_{j} \neq \Omega_{j}-\omega_{j}}\left|C_{j}\right|=0$. In particular, it will fail if $\theta \equiv 1$ and $f_{i}=$ $\Omega_{i}-\omega_{i}$ for all $1 \leq i \leq t$. In this case we can use Theorem 2 to quickly deduce a local form of a Rényi-type result. In Theorem 8, we will give the details of such a result in the special case $K=\mathbb{Q}(i)$.

(iv) A non-localized result that is, basically, a result on $v_{\mathbf{k}}(x, 0,1 / 2)$ is given in [23], but see also Theorem 9.4 of [19]. An asymptotic form of the error term for this non-localized result is given in [12].

(v) If $K=\mathbb{Q}, L=\mathbb{Q}(i), \Gamma=\{1\}$ and

$$
f\left(p^{r}\right)= \begin{cases}1 & \text { if either } p=2, p \equiv 1(\bmod 4) \text { or } 2 \mid r \text { and } p \equiv 3(\bmod 4), \\ 0 & \text { otherwise }\end{cases}
$$

then we deduce the full asymptotic version of Corollary 6 of [5]. This is a result on the integers in small intervals that are the sum of two squares and have a given number of prime factors. In this way we can see that a factor $1 /(k-1)$ ! is missing from the main term in Corollary 6 of [5].

(vi) Just as the coefficients $c_{j}(\mathbf{z}), j \geq 1$ in (2) are not what we expect when $\ell$ is close to $1 / 2$, the polynomials $P_{j, \mathbf{k}}(y), j \geq 1$, in (20) are not what would be expected for such $\ell$. In fact, if we take $\theta \equiv 1$, define $\nu_{\mathbf{k}}(x)=\#\{\mathfrak{a} \in$ $I: N \mathfrak{a} \leq x, \mathbf{f}(\mathfrak{a})=\mathbf{k}\}$ and $\delta_{\mathbf{k}}(x, \psi, \ell)=x^{-1} \nu_{\mathbf{k}}(x)-x^{-1}(2 \ell)^{-n_{K}} \nu_{\mathbf{k}}(x, \psi, \ell)$ then it can be shown, subject to the conditions on $\ell$ in Theorem 1 , that $\delta_{\mathbf{k}}$ has an expansion of the form (20) but with the sum starting at $j=1$ as opposed to $j=0$. Further, by a method of Kátai [13], it can be shown that, for such $\ell$, we have

$$
\sum_{\mathbf{k} \in \mathbb{N}_{0}^{t}} \delta_{\mathbf{k}}^{2}(x, \psi, \ell) \ll(\log x)^{-4+2 \alpha} \prod_{\substack{f_{j} \neq \Omega_{j}-\omega_{j} \\ \delta_{j} \neq 0}}\left(2 \delta_{j} \log \log x\right)^{-1 / 2} .
$$

The proof is not given here. This result could have been used to deduce Theorem 4 from a similar result for $\nu_{\mathbf{k}}(x)$. Such a result, but only with 
$t=1$, is given in Theorem 3 of [29].

3.1 Irreducible integers Let $\Gamma=C_{K}$, the ideal class group of $K$, and define the totally multiplicative function $\Theta(\mathfrak{p})=C_{i}$ if $\mathfrak{p} \in C_{i}$. Then by class field theory $\Theta$ is Frobenius multiplicative relative to $H(K) / K$, where $H(K)$ is the Hilbert Class field of $K$. Also,

$$
\Omega_{i}(\mathfrak{a})=\sum_{\substack{\mathfrak{p}^{r} \| \mathfrak{a} \\ \mathfrak{p} \in C_{i}}} r
$$

a counting function seen in [23], but see also [12], or Theorem 9.5 of [19]. From any of these references we see that we can count the irreducible integers $\alpha \in K$ satisfying $(\alpha) \in \mathcal{S}(x, \psi, \ell)$ by summing $v_{\mathbf{k}}(x, \psi, \ell)$ over $\mathbf{k}$ for which $C_{1}^{k_{1}} C_{2}^{k_{2}} \ldots C_{h}^{k_{h}}$ equals $E$, the principal class, and $C_{1}^{e_{1}} C_{2}^{e_{2}} \ldots C_{h}^{e_{h}}$, for $0 \leq e_{i} \leq k_{i}$ equals $E$ if, and only if, either all $e_{i}=0$ or $e_{i}=k_{i}$ for all $i$. Such a collection of $\mathbf{k}$ is finite and so we can sum (20), with $\theta \equiv 1$, noting that $H(K) / K$ is, like the ideal class group, abelian. Thus we deduce the result that was the initial motivation for this paper.

THEOREM 7 With $x^{-5(1-\varepsilon) / 12 n_{K}}<\ell<\exp (-R(x))$,

$$
\sum_{\substack{(\alpha) \in \mathcal{S}(x, \psi, \ell) \\ \alpha \text { irreducible }}} 1=\frac{x(2 \ell)^{n_{K}}}{\log x} \sum_{0 \leq j \leq J(x)} \frac{Q_{j}(\log \log x)}{(\log x)^{j}}+O\left(x \ell^{n_{K}} \exp (-R(x))\right),
$$

where $J(x)=\kappa_{2}(\log x)^{1 / 3}(\log \log x)^{-4 / 3}$. The polynomials $Q_{j}(X)$ are of degree at most $D-1$, where $D=D(H(K))$ is the Davenport constant of the class group $H(K)$.

4 Results in $\mathbb{Q}(i)$ In $\mathbb{Q}(i)$ the regions $\mathcal{S}(x, \psi, \ell)$ are easy to visualise as sectors of annuli. These regions can be combined together to give regions such as

$$
\mathcal{D}(z, r)=\{w \in \mathbb{C}:|w-z|<r\},
$$

the open disc with centre $z$ and radius $r$. For instance, Corollary 11(iii) of [5] states that, for fixed $k \in \mathbb{N}$ and $|z| \geq r(z) \geq|z|^{7 / 12+\varepsilon}$, we have

$$
\#\{\alpha \in \mathcal{D}(z, r): \omega(\alpha)=k\}=4 r^{2}(z) \frac{\left(\log \log \left(|z|^{2}\right)\right)^{k-1}}{(k-1) ! \log \left(|z|^{2}\right)}(1+o(1)) .
$$


(There is an error in [5] in that 4 is replaced by an erroneous $\pi$ and we have $|z|$ in the earlier paper in place of $|z|^{2}$.) Using Theorem 6, it is possible to given an expansion of the form (20) in place of (29). Here we will indicate what other results we can give in $\mathbb{Q}(i)$.

THEOREM 8 Throughout $z \in \mathbb{C}$ satisfies $|z| \geq 10$.

(i) For $k \geq 1$, and $|z|^{1 / 2} \exp (-R(|z|))<r(z)<|z|$,

$$
\begin{aligned}
\#\{\alpha \in \mathcal{D}(z, r(z)): \Omega(\alpha)-\omega(\alpha)=k\}= & 4 d_{k} r^{2}(z) \\
& +O\left(r^{8 / 5}(z)|z|^{1 / 5} \exp (-R(|z|)),\right.
\end{aligned}
$$

where

$$
\sum_{k=0}^{\infty} d_{k} z^{k}=\rho_{\mathbb{Q}(i)} \zeta_{\mathbb{Q}(i)}^{-1}(2) \prod_{\pi}\left(\frac{1-z /(1+N \pi)}{1-z / N \pi}\right) .
$$

(ii) For $|z|>r(z)>|z|^{7 / 12+\varepsilon}$,

$$
\begin{aligned}
& \frac{1}{\# \mathcal{D}(z, r)} \#\{\alpha\left.\in \mathcal{D}(z, r(z)): \omega(\alpha) \leq \log \log \left(|z|^{2}\right)+\lambda \sqrt{\log \log \left(|z|^{2}\right)}\right\} \\
& \rightarrow \Phi(\lambda)
\end{aligned}
$$

as $|z| \rightarrow \infty$.

(iii) Given integers $e \geq 2, f \geq 1$ set $\widehat{\mathbf{k}}=(e, f)$. Then for all $|z| \exp (-R(|z|))$ $>r(z)>|z|^{7 / 12+\varepsilon}$

$$
\begin{aligned}
& \#\{\alpha \in \mathcal{D}(z, r(z)): \omega(\alpha)=e, \Omega(\alpha)=e+f\} \\
& =\frac{4 r^{2}(z)}{\log \left(|z|^{2}\right)} \sum_{0 \leq j \leq J} \frac{P_{j, \widehat{\mathbf{k}}}\left(\log \log \left(|z|^{2}\right)\right)}{\left(\log \left(|z|^{2}\right)\right)^{j}} \\
& \quad+r^{2}(z) E_{\widehat{\mathbf{k}}, J}(|z|)+O\left(r^{2}(z) \exp (-R(|z|))\right)
\end{aligned}
$$

subject to $J<2 c_{0} \log |z|$. The main term is

$$
\frac{2 r^{2}(z)}{(e-2) !} P(f) \frac{(\log \log |z|)^{e-2}}{\log |z|},
$$

where $P(f)=\sum_{\pi}^{\prime}(N \pi)^{-(f+1)}$, the sum being over unassociated primes of $\mathbb{Q}(i)$. 
PROOF Define

$$
\mathcal{D}_{0}(z, r(z))=\left\{(y, \psi):\left|y^{1 / 2} e^{2 \pi i \psi / 4}-z\right|<r(z)\right\}
$$

Then Proposition 10 of [5] gives, for any $0 \leq \ell \leq 1 / 2$,

$$
\begin{gathered}
\sum_{\substack{\alpha \in \mathcal{D}(z, r) \\
C(\alpha)}} 1=\frac{1}{(2 \ell)^{2}}(1+O(\ell)) \int_{\mathcal{D}_{0}(z, r(z))}\left(\sum_{\substack{(\alpha) \in \mathcal{S}(y, \psi, \ell) \\
C(\alpha)}} 1\right) \frac{d y}{y} d \psi \\
+O(r(z)|z| \ell),
\end{gathered}
$$

where $C(\alpha)$ represents conditions on $\alpha$. If $C(\alpha)$ represents $\Omega(\alpha)-\omega(\alpha)=k$, then we can feed in the result from Theorem 2 to get

$$
\begin{gathered}
\frac{1}{(2 \ell)^{2}}(1+O(\ell)) \int_{\mathcal{D}_{0}(z, r(z))}\left\{(2 \ell)^{2} d_{k} y+O\left(y^{5 / 6} \ell^{4 / 3} \exp (-R(y))\right\} \frac{d y}{y} d \psi\right. \\
+O(r(z)|z| \ell),
\end{gathered}
$$

for any $\ell=\ell(z)>|z|^{-1 / 2} \exp (-R(|z|))$. With the observation that

$$
\iint_{\mathcal{D}_{0}(z, r(z))} d y d \psi=\frac{4}{\pi} \int_{s^{2}+t^{2}<r^{2}(z)} d s d t=4 r^{2}(z)
$$

and the choice $\ell(z)=r^{3 / 5}(z)|z|^{-4 / 5}$ we get the result stated in (i).

For (ii), the condition $C(\alpha): \omega(\alpha) \leq \log \log \left(|z|^{2}\right)+\lambda \sqrt{\log \log \left(|z|^{2}\right)}$ depends on $z$. We also choose $\ell=\ell(z)=|z|^{-5 / 12+\varepsilon / 2}$, a function of $z$. Nonetheless, the proof of Theorem 5 will give an estimate for the integrand of (31) for all $(y, \psi) \in \mathcal{D}_{0}(z, r(z))$. The result of Theorem 5 shows that the main term of (31) is asymptotically

$$
c_{0}(\mathbf{1}) \iint_{\mathcal{D}_{0}(z, r(z))} \Phi(\lambda) d y d \psi=\pi r^{2} \Phi(\lambda) \sim \# \mathcal{D}(z, r) \Phi(\lambda)
$$

as $|z| \rightarrow \infty$, using the fact that $c_{0}(\mathbf{1})=\rho_{\mathbb{Q}(i)}=\pi / 2$. For the error term of (31), we require that $r(z)|z| \ell / r^{2}(z) \rightarrow 0$ as $|z| \rightarrow \infty$. With our choice of $\ell$, this is satisfied by $|z|>r(z)>|z|^{7 / 12+\varepsilon}$. 
(iii) We need to estimate

$$
\#\{(\alpha) \in \mathcal{S}(y, \psi, \ell): \omega(\alpha)=e, \Omega(\alpha)=e+f\} .
$$

This can be done in general enforcing conditions simultaneously on $\omega_{i}$ and $\Omega_{i}$ for each $1 \leq i \leq t$. Use the notation that $\widehat{\mathbf{v}}$ will denote a vector with $2 t$ co-ordinates while $\mathbf{v}$ will consist of the first $t$ co-ordinates of $\widehat{\mathbf{v}}$. We replace f by $\widehat{\mathbf{f}}$ defined as $\widehat{\mathbf{f}}_{i}=\Omega_{i}$ or $\omega_{i}$ when $1 \leq i \leq t$, while $\widehat{\mathbf{f}}_{i}=\Omega_{i-t}-\omega_{i-t}$ for $t+1 \leq i \leq 2 t$. (In fact it would suffice if $\widehat{\mathbf{f}}_{i}=\omega_{i}$ when $1 \leq i \leq t$.)

Following the proof of Theorem 1, we find that $\widehat{\mathbf{z}} \widehat{\mathbf{f}}^{(\mathfrak{p})}=\mathbf{z}^{\mathbf{f}(\mathfrak{p})}$, so that the $n=1$ term of $U_{\mathfrak{p}}(s, \overrightarrow{\mathbf{m}}, \widehat{\mathbf{z}})$ is the same as that of $U_{\mathfrak{p}}(s, \overrightarrow{\mathbf{m}}, \mathbf{z})$. This means that the integrand of $(7)$ contains $F(s, \overrightarrow{\mathbf{0}}, \widehat{\mathbf{z}})=(s-1)^{-\alpha(\mathbf{z})} H(s, \widehat{\mathbf{z}})$, where $\alpha(\mathbf{z})=\sum_{i=1}^{t} \delta_{i} z_{i}$ and $H(s, \widehat{\mathbf{z}})$ is analytic when the coordinates of $\widehat{\mathbf{z}}$ satisfy the conditions of Theorem 1 and $|s-1| \leq 3 c_{0}$. Then, in the proof of Theorem 6 , the integrand of (21) will now contain $F^{*}(1-r, \widehat{\mathbf{z}}) r^{-\alpha(\mathbf{z})}$, where $F^{*}(s, \widehat{\mathbf{z}})=(\pi s)^{-1} H(s, \widehat{\mathbf{z}}) \sin (\pi \alpha(\mathbf{z}))$. The details of the derivation of a result for $v_{\widehat{\mathbf{k}}}(x, \psi, \ell)$, similar to $(20)$, are the same as before and valid for the same range of $\ell$.

Returning to the particular case of $(33)$ in $\mathbb{Q}(i)$, we note that we are enforcing a condition on every component of $\widehat{\mathbf{k}}$. In the notation of section 2.3 , we have $\mathcal{H}=\{1, \ldots, 2 t\}$, and so $\alpha=0$. As in part (ii), though we choose $\ell=|z|^{-5(1-\varepsilon) / 12}$, a function of $z$, we can give a result for (33), valid for all $(y, \psi) \in \mathcal{D}_{0}(z, r(z))$. Inserting such a result into (31), we attempt to write the expansion for (33) in terms of $|z|^{2}$ instead of $y$. For notational simplicity set $u=|z|^{2}$, when we find that $|y-u| \ll|z| r(z)$. Then, for each term in the expansion, we use the mean value result in

$$
\left|\frac{\left(\log _{2} y\right)^{k}}{(\log y)^{j}}-\frac{\left(\log _{2} u\right)^{k}}{(\log u)^{j}}\right| \ll(m+j) \frac{r(z) \log \log ^{m} u}{|z| \log ^{j+1} u} .
$$

We can deal with this upper bound as we did the error in (25), summing over $0 \leq m \leq p, 0 \leq p \leq e$ and $1 \leq j \leq J+1$. Then, as long as $J+2<c_{0} \log u$, we get a contribution to the integral in $(31) \ll(r(z) \log \log u) /(|z| \log u)$. 
This estimate, along with all other terms in our expansion, can be taken out of the integral in (31) on which we then use (32). It is then easily seen how all the terms in (30) arise. In addition we also have the errors

$$
\ll \frac{r^{3}(z) \log \log |z|}{|z| \log |z|}+\frac{\ell r^{2}(z)}{\log |z|}+r(z)|z| \ell,
$$

where the second one comes from the $O(\ell)$ factor in (31). We see these terms are sufficiently small subject to the conditions on $r(z)$. For the main term, the coefficients of $P_{0,(e, f)}$ are constructed from the $\beta_{0, \widehat{\mathbf{u}}}$, defined by

$$
\sum_{\widehat{\mathbf{u}}} \beta_{0, \widehat{\mathbf{u}}} \widehat{\mathbf{z}}^{\widehat{\mathbf{u}}}=F^{*}(1, \widehat{\mathbf{z}})=\frac{\sin \left(\pi z_{1}\right)}{\pi} \rho_{\mathbb{Q}(i)}^{z_{1}} \prod_{\pi}\left(1+\sum_{n \geq 1} \frac{z_{1} z_{2}^{n-1}}{N \pi^{n}}\right) .
$$

We immediately see that if $\beta_{0, \widehat{\mathbf{u}}} \neq 0$ for some $u=\left(u_{1}, u_{2}\right)$ with $u_{2} \neq 0$, then

$u_{1} \geq 2$. Further, $\beta_{0,(2, f)}=\sum_{\pi}^{\prime}(N \pi)^{-(f+1)}$. Working through the proof of Theorem 6 , we obtain the main term quoted.

Note (vii) In general the degree of the polynomial in the main term for $v_{\widehat{\mathbf{k}}}(x, \psi, \ell)$ is $\leq \sum_{1}^{t} k_{i}-(s+1)$, where $s$ is the number of non-zero $\widehat{k}_{i}, t+1 \leq i \leq 2 t$.

Part (ii) of Theorem 8 generalises the result for short intervals of $\mathbb{Q}$ due to Kátai, [13]. Part (iii) generalises the non-localized version in $\mathbb{Q}$ due to Delange, [6].

\section{Acknowledgments}

This paper was partly written while the author enjoyed the hospitality and excellent working conditions of the Centre de recherches Mathématiques at the Université de Montréal.

\section{References}

[1] M. D. Coleman, The distribution of points at which binary quadratic forms are prime, Proc. London Math. Soc (3), 61 (1990), 433-456.

[2] M. D. Coleman, A zero-density region for the Hecke L-functions, Mathematika 37 (1990) 287-304 
[3] M. D. Coleman, The Rosser-Iwaniec sieve in number fields with an application, Acta Arith. 65 (1993) 53-83.

[4] M. D. Coleman, Relative norms of prime ideals in small regions, Mathematika 43 (1996), 40-62.

[5] M. D. Coleman, The Hooley-Huxley contour method for problems in number fields I: Arithmetic Functions, J. Number Theory, 74, (1999), 250277.

[6] H. Delange, Sur des formules de Alte Selberg, Acta Arith. 19 (1971), 105-146.

[7] H. Delange, Sur un théorème de Rényi III, Acta Arith 23 (1973) 153-182

[8] E. Fogels, On primes representable by a binary quadratic form, Latvijas PSR Zinatnu Akad. Vestis Fiz. Tehn. Zinatnu Ser. 1, (1966), 71-79.

[9] E. Hecke, Eine neue Art von Zeta Functionen und ihre Beziehungen zur Verteilung der Primzahlen I, II Math Z. 1 (1918), 357-376; 6 (1920), 11-51.

[10] M. N. Huxley, On the difference between consecutive primes. Invent. Math. 15 (1972), 164-170.

[11] G. J. Janusz, Algebraic number fields (Academic Press, 1973).

[12] J. Kaczorowski, Some remarks on factorization in algebraic number fields, Act Arith. 43 (1983), 53-68.

[13] I. Kátai, A remark on a paper of K. Ramachandra, Lecture Notes in Math. 1122 (1985), 147-152.

[14] J. P. Kubilius, On some problems of the geometry of prime numbers, Mat. Sb. (N.S.) 31 (1952), 791-794.

[15] J. P. Kubilius, On a problem in the n-dimensional analytic theory of numbers, Viliniaus Valst. Univ. Mokslo dardai Fiz. Chem. Moksly Ser. 4 (1955), 5-43.

[16] J. C. Lagarias, A. M. Odlyzko, Effective versions of the Chebotarev density theorem, Algebraic number fields, ed. A. Frölich, (Academic Press, 
1977).

[17] E. Landau, Handbuch der Lehre der Primzahlverteilung Bd 2, S. 643, Teubner, Leipzig, 1909.

[18] S. Lang, Algebraic number theory, 2nd ed. (Springer-Verlag, 1994).

[19] W. Narkiewicz, Elementary and analytic theory of algebraic numbers, PWN Warszawa 1974.

[20] R. W. K. Odoni, The Farey density of norms of subgroups of global fields (I), Mathematika, 20 (1973), 155-169.

[21] R. W. K. Odoni, Some global norm density results obtained from an extended Cebotarev density theorem, Algebraic number fields, ed. A. Frölich, (Academic Press, 1977).

[22] K. Ramachandra, Some problems of analytic number theory, Acta Arith. 31 (1976) 313-324.

[23] J. P. Rémond, Evaluations asymptotiques dans partitions certaines semi-groupes, C. R. Acad. Sci. Paris 260 (1965) 2250-2251; 262 (1966) A271-A273.

[24] J. -P. Serre, Divisibilité de certaines fonctions arithmétiques, L'Enseignement Math (II) XXII (3/4) (1976), 227-260.

[25] A. V. Sokolovskii, A theorem on the zeros of Dedekind's zeta-function and the distance between "neighboring" prime ideals. Acta Arith. 13 (1967/1968), 321-334.

[26] G. Tenenbaum, Introduction to analytic and probabilistic number theory (Cambridge University Press 1995).

[27] D. Wolke, On a problem of A. Rényi, Mh. Math, 111, (1991) 323-330.

[28] J. Wu, Sur un probleme de Rényi, Mh Math 117 (1994), 303-322.

[29] J. Wu, A sharpening of effective formulas of Selberg-Delange type for some arithmetic functions on the semigroup $G_{K}$, J. Number Theory $\mathbf{5 9}$ (1996), 1-19. 